\title{
Proton receptors regulate synapse-specific reconsolidation in the amygdala
}

2 Erin E Koffman ${ }^{1,2 \#}$, Charles M Kruse ${ }^{1,2 \#}$, Kritika Singh ${ }^{2}$, FarzanehSadat Naghavi ${ }^{2}$,

3 Jennifer Egbo ${ }^{2}$, Sandra Boateng ${ }^{2}$, Mark Houdi BA ${ }^{2}$, Boren Lin ${ }^{2}$, Jacek Debiec ${ }^{3}$, Jianyang Du ${ }^{1,4^{*}}$

$5{ }^{1}$ Department of Anatomy and Neurobiology, University of Tennessee Health Science Center, Memphis, TN 38163, USA.

${ }^{3}$ Molecular \& Behavioral Neuroscience Institute and Department of Psychiatry, University of Michigan, Ann Arbor, MI, USA.

${ }^{4}$ Neuroscience Institute, University of Tennessee Health Science Center, Memphis, TN, United States.

13 \#Equal contribution

\section{${ }^{*}$ Correspondence:}

16 Jianyang Du

17 Department of Anatomy and Neurobiology,

18 University of Tennessee Health Science Center,

19 Memphis, TN 38163, USA

20 TEL: 901-448-3463

21 E-MAIL: jdu15@uthsc.edu 


\section{SUMMARY}

24 When an extinction procedure is performed within the reconsolidation window, the

25 original aversive memory can be replaced by one that is less traumatic. Recent studies

26 revealed that carbon dioxide $\left(\mathrm{CO}_{2}\right)$ inhalation during retrieval enhances memory lability.

27 However, the effects of $\mathrm{CO}_{2}$ inhalation on the central nervous system can be extensive,

28 and there is lack of evidence suggesting that the effects of $\mathrm{CO}_{2}$ are selective to a

29 reactivated memory. We discovered that $\mathrm{CO}_{2}$ inhalation paired with memory retrieval

30 potentiates the specific aversive memory trace, resulting in greater memory lability. The

31 specific effects of $\mathrm{CO}_{2}$ depend on acid-sensing ion channels (ASICs), the proton

32 receptors that are involved in synaptic transmission and plasticity in the amygdala. In

33 addition, $\mathrm{CO}_{2}$ inhalation alters memory lability via synaptic plasticity at selectively

34 targeted synapses. Overall, our results suggest that inhaling $\mathrm{CO}_{2}$ during the retrieval

35 event increases the lability of an aversive memory through a synapse-specific

36 reconsolidation process.

38 KEYWORDS: carbon dioxide $\left(\mathrm{CO}_{2}\right)$; acid-sensing ion channels; proton receptors;

39 aversive conditioning; aversive memory; memory retrieval; AMPA receptors;

40 reconsolidation; synaptic plasticity; spine structure. 


\section{INTRODUCTION}

48 Recently, aversive memory research in both rodents and humans has focused on a

49 window of time after aversive memory retrieval, in which the memory is labile and

50 subject to intervention (Kida, 2020; Lee, 2009; Monfils et al., 2009; Nader et al., 2000;

51 Sara, 2000; Schiller et al., 2010; Tronson and Taylor, 2007). This window of time is

52 known as a reconsolidation window, and is thought to last up to six hours after initiation

53 (Clem and Huganir, 2010; Duvarci and Nader, 2004; Monfils et al., 2009; Schiller et al.,

54 2010). Several studies have demonstrated that interrupting the updating process

55 aroused by retrieval prevents memory restorage, generating selective amnesia (Agren

56 et al., 2012; Clem and Huganir, 2010; Mactutus et al., 1979; Monfils et al., 2009; Nader

57 et al., 2000; Schiller et al., 2010). Studies using rodent models have indicated that

58 pharmacological intervention within the reconsolidation window successfully erases

59 reactivated specific aversive memory (Nader et al., 2000; Sara and Hars, 2006; Schiller

60 et al., 2010). Despite the efficacy, ethical and practical concerns may prevent similar

61 pharmacological interventions from being used in humans (Agren et al., 2012; Schiller

62 et al., 2010). Recently, drug-free paradigms have been proposed that effectively prevent

63 the return of aversive memories in both rodents and humans (Bjorkstrand et al., 2016;

64 Clem and Huganir, 2010; Huang et al., 2020; Liu et al., 2014; Monfils et al., 2009;

65 Schiller et al., 2010). Monfils et al. developed a novel protocol in which an isolated

66 retrieval trial was followed by an extinction event within a specific time frame. This

67 resulted in the weakening of the original memory trace, thereby preventing reversion of

68 the original aversive memory by spontaneous recovery, renewal, or reinstatement

69 (Clem and Huganir, 2010; Jarome et al., 2015; Monfils et al., 2009; Quirk et al., 2010; 
70 Wu et al., 2017). These studies suggest the mechanism by which retrieval changes the

71 lability of memory may become a target for novel clinical treatments for anxiety

72 disorders.

74 Currently, the outcomes of the combination of retrieval and extinction paradigms are

75 variable, depends on multiple factors, in both rodents (Auber et al., 2013; Chan et al.,

76 2010; Costanzi et al., 2011; Goode et al., 2017; Ishii et al., 2012) and humans (Golkar

77 et al., 2012; Klucken et al., 2016). Because it is difficult to completely erase the original

78 memory, a more reliable strategy for triggering memory erasure is necessary. In search

79 of this strategy, we studied the effects that $\mathrm{CO}_{2}$ inhalation may have on the erasure of a

80 specific memory. As we showed previously, when mice receive a retrieval tone

81 supplemented by $\mathrm{CO}_{2}$ inhalation, the memory becomes more labile than the

82 presentation of a retrieval tone alone (Du et al., 2017). Within the reconsolidation

83 window, the labile memory becomes more convertible, either weakened by extinction or

84 strengthened by reconditioning. Moreover, the effects of $\mathrm{CO}_{2}$ inhalation on memory

85 lability were dependent on acid-sensing ion channels (ASICs), since disrupting ASICs in

86 the amygdala eliminated these effects of $\mathrm{CO}_{2}$ (Du et al., 2017).

88 Recent studies have revealed that protons are potential neurotransmitters (Du et al.,

89 2014; Highstein et al., 2014; Kawasaki et al., 2009) and ASICs serve as postsynaptic

90 proton receptors that play key roles in neurotransmission and synaptic plasticity in the

91 amygdala, a brain region that is critical for the formation of aversive memories (Cohen

92 et al., 2017; Du et al., 2014; Farley et al., 2018; Ziemann et al., 2009). ASICs are

93 members of the Degenerin/ Epithelial sodium channel (DEG/ ENaC) family (Ben- 
94 Shahar, 2011). To date, six family members have been identified (ASIC1a, ASIC1b,

95 ASIC2a, ASIC2b, ASIC3, and ASIC4) (Wemmie et al., 2013). These proteins assemble

96 as homo- or heterotrimers to form channels that are proton-gated, voltage-insensitive,

97 permeable to both $\mathrm{Na}^{+}$and $\mathrm{Ca}^{2+}$ and activated by extracellular protons (Waldmann et al.,

98 1997; Waldmann and Lazdunski, 1998). ASIC1a is widely expressed in many areas of

99 the brain, where it is associated with numerous brain functions and disorders, including

100 hippocampally-dependent learning and memory, anxiety, depression, stroke,

101 neurodegeneration, seizure, inflammation, and nerve injury (Chu and Xiong, 2012; Gao

102 et al., 2005; Ortega-Ramírez et al., 2017; Wang et al., 2018; Wemmie et al., 2002;

103 Wemmie et al., 2006). ASIC1a is highly expressed in the amygdala and its ion channel

104 activity is evoked by a reduction in extracellular $\mathrm{pH}$ within the physiological range

105 (Taugher et al., 2017; Wemmie et al., 2003). Disruption of ASIC1a affects synaptic

106 transmission and plasticity (Mango and Nisticò, 2019; Soto et al., 2018; Wemmie et al.,

107 2003; Wu et al., 2004). Loss of ASIC1a function also leads to impaired high-frequency

108 electrical stimulation-induced long-term potentiation (LTP) (Chiang et al., 2015; Du et

109 al., 2014; Liu et al., 2016; Wemmie et al., 2002). Moreover, we demonstrated that

110 presynaptic stimulation induces a transient synaptic drop in $\mathrm{pH}$ and activates ASIC-like

111 excitable postsynaptic currents (EPSCs) in pyramidal neurons (Du et al., 2014; Kreple

112 et al., 2014), suggesting that protons sufficiently activate postsynaptic ASIC1a in

113 synaptic transmission. In mice, disruption of ASIC1a activity reduces innate fear

114 learning and memory and alters neuronal activity in the fear circuit (Coryell et al., 2007),

115 whereas its overexpression has opposite effects (Wemmie et al., 2004). Also, reducing

$116 \mathrm{pH}$ by $\mathrm{CO}_{2}$ inhalation or the injection of acid into the amygdala induced aversive 
117 behavior response and enhanced aversive memory, and the $\mathrm{CO}_{2}$ effects are ASIC1a

118 dependent (Ziemann et al., 2009).

120 Although our previous data suggested that $\mathrm{CO}_{2}$ inhalation affects the aversive memory

121 retrieval and alters the memory lability, we also know that the $\mathrm{CO}_{2}$ - affected memory

122 trace is associated with the retrieval cue (Du et al., 2017), inhalation of $\mathrm{CO}_{2}$ may reduce

$123 \mathrm{pH}$ outside of the amygdala (Dulla et al., 2005; Zandbergen et al., 1989). Thus, whether

$124 \mathrm{CO}_{2}$ and ASICs specifically regulate memory trace in retrieval is still unknown and this

125 question is outstanding. In this study, we found that $\mathrm{CO}_{2}$ inhalation paired with memory

126 retrieval selectively potentiated memory lability in mice. Furthermore, electrorheological

127 and imaging studies in brain slices support the conclusion that the effects of $\mathrm{CO}_{2}$ on

128 memory retrieval are specifically associated with a given memory. Our study proposes

129 that inhaling $\mathrm{CO}_{2}$ within the reconsolidation window regulates aversive memory with

130 specificity, providing a unique angle to further study the mechanism by which memory is

131 modulated.

133 EXPERIMENTAL PROCEDURES

134 Mice

135 For our experiment, we used both male and female mice between 10-14 months of age.

136 Mice were derived from a congenic C57BL/6 background including wild-type, ASIC1a

137 knock out (ASIC1 $\left.\mathrm{a}^{-/-}\right)$, and TetTag-c-fos-tTA mice. TetTag-cFos-tTA mice were

138 obtained from Jackson Laboratory and crossed with C57BL/6J mice. Mice carrying

139 the Fos-tTA transgene were selected; Fos-tTA mice have a Fos promoter driving

140 expression of nuclear-localized, two-hour half-life EGFP (shEGFP) (Du et al., 2017; 
141 Koffman and Du, 2017; Ramirez et al., 2013). The Fos promoter also drives the

142 expression of tetracycline transactivator (tTA), which bind to the tetracycline-responsive

143 element (TRE) site on an injected recombinant adeno-associated virus, AAV2/9-TRE-

144 mCherry virus, resulting in the expression of mCherry (Du et al., 2017; Koffman and Du,

145 2017). The binding of the tTA to the TRE site is inhibited by doxycycline (DOX).

146 Inhibition of tTA binding prevents target gene expression (Das et al., 2016; Liu et al.,

147 2012; Ramirez et al., 2013).

149 Both male and female mice age10-14 weeks were randomly selected for the experiment

150 groups. Experimental mice were maintained on a standard 12-hour light-dark cycle and

151 received standard chow and water ad libitum. Animal care and procedures met the

152 National Institutes of Health standards. The University of Tennessee Health Science

153 Center Laboratory Animal Care Unit (Protocol \#19-0112) and University of Toledo

154 Institutional Animal Care and Use Committee (Protocol \#108791) approved all

155 procedures.

Aversive conditioning, retrieval, extinction, and memory test

158 The protocols for each experiment are detailed in the schematics of each figure. All

159 mice were handled by experimenters for 30 minutes on each of 3 days before aversive

160 conditioning. On day 1 , mice were habituated to the aversive conditioning chamber

161 (Med Associates Inc.) for 7 minutes. Mice were then exposed to varying conditioning

162 protocols, as described below. 
164 Experiment 1: Standard conditioned stimulus (CS) auditory aversive conditioning,

165 retrieval, extinction, and memory tests.

166 On day 1 in a curated environment (context $A$ ), the experimental mice were presented

167 with six pure tones ( $80 \mathrm{~dB}, 2 \mathrm{kHz}, 20$ seconds each) paired with 6 foot shocks - one

168 shock at the end of each tone $(0.7 \mathrm{~mA}, 2$ seconds). The interval between each tone was

169100 seconds. On day 2, the mice were placed into a new environment (context B) and

170 habituated for 4 minutes. Mice then inhaled either unaltered air or air containing $10 \%$

$171 \mathrm{CO}_{2}$ for 7 minutes. Five minutes after inhalation of $\mathrm{CO}_{2}$ or air began, mice were

172 presented with one 20 second pure tone to retrieve the memory. The mice were then

173 returned to their home cages. 30 minutes later, the mice returned to the retrieval

174 chamber (context B) and underwent two rounds of extinctions. In the first round of

175 extinction, mice were exposed to 20 pure tones with an interval between tones of 100

176 seconds. Mice were then returned to their home cage. 30 minutes later, the mice went

177 through the extinction protocol again with 20 pure tones. On day 7, the mice were tested

178 to see if their aversive response would recur via spontaneous recovery in context B with

1794 pure tones. Thirty minutes after spontaneous recovery, the mice were returned to the

180 original context of the aversive memory, context A, in a recovery protocol with 4 pure

181 tones.

182

183 Freezing behavior in mice (the absence of movement beyond respiration) is used as a

184 measure of aversive response. To evaluate the outcomes of freezing behavior in mice,

185 the percentage of time during CS presentation spent in freezing was scored

186 automatically using VideoFreeze software (Med Associates Inc.). In the spontaneous 
187 recovery and renewal tests, outcomes of the percentage of time freezing were averaged

188 from each of the 4 CSs.

Experiment 2: Two distinct CSs aversive conditioning, retrieval, extinction, and

191 memory tests.

192 This procedure was used to test the specificity of the effects of $\mathrm{CO}_{2}$ on memory

193 retrieval. The context settings and parameters are similar to the above standard one CS

194 auditory aversive conditioning. In contrast to experiment 1, the mice were presented

195 with three pure tones ( $80 \mathrm{~dB}, 2 \mathrm{kHz}, 20$ seconds each) that alternated with three white

196 noises (60 dB, 2 kHz, 20 seconds each); all six stimuli were paired with foot shocks. On

197 day 2 , the mice inhaled either unaltered air or air containing $10 \% \mathrm{CO}_{2}$ for 7 minutes.

198 Five minutes after inhalation of $\mathrm{CO}_{2}$ or air began, the mice underwent retrieval with one

199 single pure tone followed by one white noise with or without $\mathrm{CO}_{2}$. This was followed

200 thirty minutes later by two sections of extinctions with either pure tones or white noises.

201 On day 7, the mice were tested via spontaneous recovery and renewal protocols with 4

202 pure tones and 4 white noises respectively.

204 Experiment 3: Two distinct CSs aversive conditioning, retrieval, anisomycin, and

205 memory tests.

206 In a series of experiments, the standard extinction procedure was replaced with

207 amygdala infusion of anisomycin (detailed in the surgery procedure below). In brief, the

208 cannula was implanted on the amygdala 4-7 days before the behavioral experiments.

209 Starting on day 1 , the mice were subjected to the aversive conditioning described in

210 experiment 2. On day 2, 30 minutes after retrieval, the mice were infused with 62.5 
$211 \mu \mathrm{g} / \mu \mathrm{l}$ anisomycin via the cannula in the lateral nuclei of the amygdala (LA) bilaterally

212 and returned to their home cage (Debiec et al., 2010). On day 7, the mice were tested

213 via spontaneous recovery and renewal as described in experiment 2.

214

215 Surgery and chemical infusion

216 For the cannula placement procedure, mice were anesthetized with isoflurane through

217 an anesthetic vaporizer, secured to the stereotaxic instrument and a cannula made from

218 a 25-gauge needle was inserted bilaterally into LA (relative to bregma: $-1.2 \mathrm{~mm}$

219 anterioposterior; $\pm 3.5 \mathrm{~mm}$ mediolateral; $-4.3 \mathrm{~mm}$ dorsoventral) (Du et al., 2017; Koffman

220 and Du, 2017). Dental cement secured the cannula and bone anchor screw in place.

221 Mice recovered for 4-5 days before any subsequent testing was carried out. A $10 \mu \mathrm{L}$

222 Hamilton syringe connected to a 30-gauge injector was inserted $1 \mathrm{~mm}$ past the cannula

223 tip to inject anisomycin (diluted in $1 \mu$ artificial cerebrospinal fluid (ACSF), pH 7.3) over

$2245 \mathrm{sec}$. The injection sites were mapped post-mortem by sectioning the brain $(10 \mu \mathrm{m}$

225 coronal) and performing cresyl violet staining.

227 Brain slice preparation and patch-clamp recording of amygdala neurons

228 Ten minutes after the memory retrieval experiment ended, mice were euthanized with

229 overdosed isoflurane and whole brains were dissected into pre-oxygenated $\left(5 \% \mathrm{CO}_{2}\right.$

230 and $95 \% \mathrm{O}_{2}$ ) ice-cold high sucrose dissection solution containing (in $\mathrm{mM}$ ): 205 sucrose,

$2315 \mathrm{KCl}, 1.25 \mathrm{NaH}_{2} \mathrm{PO}_{4}, 5 \mathrm{MgSO}_{4}, 26 \mathrm{NaHCO}_{3}, 1 \mathrm{CaCl}_{2}$, and 25 glucose (Du et al., 2017).

232 A vibratome sliced brains coronally into $300 \mu \mathrm{m}$ sections that were maintained in normal

233 ACSF containing (in mM): $115 \mathrm{NaCl}, 2.5 \mathrm{KCl}, 2 \mathrm{CaCl}_{2}, 1 \mathrm{MgCl}_{2}, 1.25 \mathrm{NaH}_{2} \mathrm{PO}_{4}, 11$

234 glucose, $25 \mathrm{NaHCO}_{3}$ bubbled with $95 \% \mathrm{O}_{2} / 5 \% \mathrm{CO}_{2}, \mathrm{pH} 7.35$ at $20^{\circ} \mathrm{C}-22^{\circ} \mathrm{C}$. Slices were 
235 incubated in the ACSF at least 1 hour before recording. For experiments, individual

236 slices were transferred to a submersion-recording chamber and were continuously

237 perfused with the $5 \% \mathrm{CO}_{2} / 95 \% \mathrm{O}_{2}$ solution $(\sim 3.0 \mathrm{ml} / \mathrm{min})$ at room temperature $\left(20^{\circ} \mathrm{C}-\right.$

$\left.23822^{\circ} \mathrm{C}\right)$

240 As we described previously (Du et al., 2017), pyramidal neurons in the lateral amygdala

241 were studied using whole-cell patch-clamp recordings. The pipette solution containing

242 (in mM): $135 \mathrm{KSO}_{3} \mathrm{CH}_{3}, 5 \mathrm{NaCl}, 10$ HEPES, 4 MgATP, $0.3 \mathrm{Na}_{3} \mathrm{GTP}, 0.5 \mathrm{~K}-\mathrm{EGTA}$

243 (mOsm=290, adjusted to $\mathrm{pH} 7.25$ with $\mathrm{KOH}$ ). The pipette resistance (measured in the

244 bath solution) was 3-5 M . High-resistance (>1G $\mathrm{G}$ ) seals were formed in voltage-

245 clamp mode. Picrotoxin $(100 \mu \mathrm{M})$ was added to the ACSF throughout the recordings to

246 yield excitatory responses. In AMPAR current rectification experiments, we applied D-

247 APV $(100 \mu \mathrm{M})$ to block NMDAR-conducted EPSCs. The peak amplitude of ESPCs was

248 measured to determine current rectification. The amplitude was measured ranging from

$249-80 \mathrm{mV}$ to $+60 \mathrm{mV}$ in $20 \mathrm{mV}$ steps. The peak amplitude of EPSCs at $-80 \mathrm{mV}$ and +60

$250 \mathrm{mV}$ was measured for the rectification index. In EPSC ratio experiments, neurons were

251 measured at $-80 \mathrm{mV}$ to record AMPAR-EPSCs and were measured at $+60 \mathrm{mV}$ to record

252 NMDAR-EPSCs. To determine the AMPAR-to-NMDAR ratio, we measured the peak

253 amplitude of ESPCs at $-80 \mathrm{mV}$ as AMPAR-currents, and peak amplitude of EPSCs at

$254+60 \mathrm{mV}$ at $70 \mathrm{~ms}$ as NMDAR-currents after onset. Data were acquired at $10 \mathrm{kHz}$ using

255 Multiclamp 700B and pClamp 10.1. The mEPSCs events (>5pA) were analyzed in

256 Clampfit 10.1. The decay time (т) of mEPSCs was fitted to an exponential using

257 Clampfit 10.1. 


\section{Immunohistochemistry and cell counting}

260 The AAV-TRE-mCherry plasmid was obtained from the laboratory of Dr. Susumu

261 Tonegawa (Liu et al., 2012; Ramirez et al., 2013), and was used to produce $A A V_{2 / 9}$ by

262 the University of lowa Gene Transfer Vector Core. For one week leading up to virus

263 microinjection, TetTag Fos-tTA mice were fed with food containing $40 \mathrm{mg} / \mathrm{kg} \mathrm{DOX.} \mathrm{We}$

264 used a $10 \mu \mathrm{l}$ Hamilton microsyringe and a WPI microsyringe pump to inject virus $(0.5 \mu \mathrm{l}$

265 of $1.45 \mathrm{E}+12$ viral genomes/ml of $\left.A A V_{2 / 9}-T R E-m C h e r r y\right)$ bilaterally into the amygdala

266 (relative to bregma: $-1.2 \mathrm{~mm}$ anterioposterior; $\pm 3.5 \mathrm{~mm}$ mediolateral; $-4.3 \mathrm{~mm}$

267 dorsoventral), as described previously (Du et al., 2017; Koffman and Du, 2017). For

268 a two-week window between surgery and behavior training, mice were housed and fed

269 with a DOX-containing diet. The DOX-containing diet was ceased twenty-four hours

270 before aversive conditioning began on day one (replaced by a regular diet), then

271 immediately restarted afterward. Thirty minutes after retrieval on day two, the mice were

272 euthanized according to protocol. We used transcardial perfusion with $4 \%$

273 paraformaldehyde (PFA) to fix whole brains, followed by continued fixation in 4\% PFA at

$2744^{\circ} \mathrm{C}$ for 24 hours (Wright et al., 2020). Following perfusion, we used a vibratome (Leica

$275 \mathrm{VT}-1000 \mathrm{~S})$ to dissect $50 \mu \mathrm{m}$ amygdala coronal slices, which were collected in ice-cold

276 PBS. To complete immunostaining, slices were placed in Superblock solution (Thermo

277 Fisher Scientific) plus $0.2 \%$ Triton X-100 for 1 hour and incubated with primary

278 antibodies (1:1000 dilution) at $4^{\circ} \mathrm{C}$ for 24 hours (Du et al., 2017). Primary antibodies we

279 used include: rabbit polyclonal IgG anti-RFP (Rockland Cat\# 600-401-379); chicken IgY

280 anti-GFP (Thermo Fisher Scientific Cat\# A10262) and mouse anti-NeuN (Millipore Cat\#

281 MAB377X) (Liu et al., 2012; Ramirez et al., 2013). We then washed and incubated 
282 slices for one hour with secondary antibodies (Alexa Fluor 488 goat anti-chicken IgG

$283(\mathrm{H}+\mathrm{L})($ Molecular Probes Cat\# A-11039); Alexa Fluor 568 goat anti-rabbit lgG $(\mathrm{H}+\mathrm{L})$

284 (Molecular Probes Cat\# A-21429); Alexa Fluor 647 goat anti-mouse IgG (H+L) (Thermo

285 Fisher Scientific Cat\# A-21235), 1:200 dilution). VectaShield H-1500 (Vector

286 Laboratories Cat\# $\mathrm{H}-1500$ ) was used to mount slices, while confocal microscopy was

287 used to view the slices. We used ImageJ software to analyze dendritic spine

288 morphology. Thin, mushroom and stubby spines were categorized based on the

289 following parameters: 1) mushroom spines: head-to-neck diameter ratio >1.1:1 and

290 spine head diameter >0.35 $\mu \mathrm{m}$; 2); thin spines: head-to-neck diameter ratio >1.1:1 and

291 spine head diameter $>0.35 \mu \mathrm{m}$ or spine head-to-neck diameter ratios $<1.1: 1$ and spine

292 length-to-neck diameter > $2.5 \mu \mathrm{m}$; 3); stubby spines: spine head-to-neck diameter ratios

$293<1.1: 1$ and spine length-to-neck diameter $\leq 2.5 \mu \mathrm{m}$ (Kreple et al., 2014; Wright et al.,

294 2020).

295

296 Statistical analysis

297 One-way ANOVA and Tukey's post-hoc multiple comparison tests were used for

298 statistical comparison of groups. An unpaired Student's t-test was used to compare

299 results between two groups. $P<0.05$ was considered statistically significant, and we did

300 not exclude potential outliers from our data except the ones did not receive successful

301 aversive conditioning. The graphing and statistical analysis software Graphpad Prism 8

302 was used to analyze statistical data, which was presented as means \pm SEM. Sample

303 sizes $(n)$ are indicated in the figure legends, and data are reported as biological

304 replicates (data from different mice, different brain slices). Each group contained tissues 
305 pooled from 4-5 mice. Due to variable behavior within groups, we used sample sizes of

306 10-16 mice per experimental group as we previously described in earlier experiments

307 (Du et al., 2017). In behavioral studies, we typically studied groups with four randomly

308 assigned animals per group, as our recording equipment allowed us to record four

309 separate animal cages simultaneously. The experiments were repeated with another set

310 of four animals until we reached the target number of experimental mice per group.

311 Experimentation groups were repeated in this manner so that each animal had the

312 same controlled environment-the same time of day and with similar handling,

313 habituation, and processes.

\section{RESULTS}

316 Our recent studies suggested that $\mathrm{CO}_{2}$ inhalation throughout memory retrieval

317 enhances the lability of the memory and boosts the efficiency of the memory erasure

318 (Du et al., 2017). To further test whether the effects of $\mathrm{CO}_{2}$ on memory retrieval are

319 synapse-specific, we designed a series of unique experiments by which we were able to

320 generate two distinct auditory aversive memories and identify the specificity of $\mathrm{CO}_{2}$

321 effects on each of them.

$323 \mathrm{CO}_{2}$ selectively enhances the lability of auditory aversive memory in the

\section{4 amygdala}

325 Previous studies have described an aversive conditioning paradigm in which memory

326 can be selectively reactivated and reconsolidated, suggesting synapse-specific

327 reconsolidation of distinct aversive memories in the amygdala (Debiec et al., 2010;

328 Doyere et al., 2007). We followed this paradigm albeit with modifications (Fig.1A, Fig. 
s1A). On day 1 , we trained the animals with two distinct conditioned stimuli: three pure

330 tones and three white noises paired with one foot-shock per stimuli as aversive

331 unconditioned stimuli (US) (see the detailed description in Materials and Methods). We

332 evaluated the outputs of the aversive conditioning through the percentage of the

333 freezing time within the time of CSs. The freezing was significantly increased after each

334 of the three conditioned stimuli, indicating the mice were trained sufficiently under the

335 designed condition (Fig.1B, Fig. s1B).

337 On day 2, the animals were placed into a new context (context B) and presented with

338 one pure tone followed by a single noise (or vice versa) to retrieve the memory (Fig. 1A,

339 C, Fig. s1A, C). The animals were then returned to their home cages. 30 minutes later,

340 all mice underwent two blocks of extinctions in context B, each extinction contains 20

341 pure tones (Fig.1D) or 20 white noise (Fig. s1D). At the end of the extinction procedure,

342 the freezing dropped down to a low level (Fig. 1D, Fig. s1D), suggesting that the

343 extinction procedure was sufficient to suppress the memory. Five days later, the mice

344 underwent spontaneous recovery (context B) and renewal (context A) respectively. Four

345 tones and four noises were presented throughout the memory test (Fig.1A, Fig. s1A).

346 The group that underwent extinction with a specific CS showed specificity in which

347 freezing response was lowered after extinction (Fig.1E, Fig. s1E). For example, when

348 the pure tone was presented during extinction, freezing in the pure tone group was

349 lower than freezing in the noise group (Fig.1E), and vice versa (Fig. s1E). When

350 retrieval was paired with $10 \% \mathrm{CO}_{2}$ inhalation, memory erasure effects were enhanced

351 (Fig.1 F-I, Fig. s1F-I), and there are statistical significances between the groups with or 
352 without $\mathrm{CO}_{2}$ in both spontaneous recovery and renewal (green columns, Fig.1 J-K and

353 red columns, Fig. s1J-K). To further evaluate the specificity of the effects of

354 reconsolidation on memory modifications, we designed another retrieval protocol in

355 which we presented pure tone and white noise, either of them paired with $10 \% \mathrm{CO}_{2}$

356 inhalation, followed by an unrelated extinction of CS, white noise, or pure tone

357 respectively (Fig. s2). Consistent with our expectation, $\mathrm{CO}_{2}$ did not boost the effects on

358 the retrieved memory in the absence of paired extinction, compared to the data in Fig. 1

359 and Fig. S1 (Fig. s2B-I). When compared to the data in Fig. 1F-I and Fig. s1F-I, we

360 found the application of $10 \% \mathrm{CO}_{2}$ to the retrieval event failed to enhance the outcome

361 after extinction, indicating a specificity of the $\mathrm{CO}_{2}$ effects. In all, our data suggest that

362 memory encoded in the amygdala can be distinct, and the effects of $\mathrm{CO}_{2}$ on memory

363 are specific.

365 To focus on testing the specific effects of $\mathrm{CO}_{2}$ on retrieval, we replaced the extinction

366 procedure with an injection of a protein synthesis inhibitor, anisomycin, to obliterate the

367 aversive memory (Fig. 2A). Anisomycin, when injected bilaterally into the amygdala

368 after retrieval, causes memory erasure compared to the saline injection group (Debiec

369 et al., 2010). We conditioned the mice and retrieve the memory with pure tones

370 (Fig. 2B-C), followed by anisomycin/saline injection (Fig. 2D). Our experiments show

371 that anisomycin disrupts the memory during reconsolidation (Fig. 2E), and that memory

372 retrieval is required for memory erasure with anisomycin injection (Fig. s3A-E).

373 Consistent with the extinction results in Fig. 1, when retrieval was paired with $10 \%$

$374 \mathrm{CO}_{2}$ inhalation, we found anisomycin reduced more aversive response-further 
375 confirming that $\mathrm{CO}_{2}$ enhances memory lability specifically (Fig. 2F-I). To exclude the

376 possibility that anisomycin associates with one CS other the other, as rigorous controls,

377 we conditioned the mice with both pure tone and white noise and carried out memory

378 retrieval with both CSs and found anisomycin has equal effects on memory in both tone

379 and noise groups (Fig. s3F-M). When $10 \% \mathrm{CO}_{2}$ was applied while the CSs were

380 presented, the retrieval group paired with $\mathrm{CO}_{2}$ showed less freezing, regardless of the

381 type of CSs (pure tone or white noise) (Fig.s4 B-I). As rigorous controls, we applied

$382 \mathrm{CO}_{2}$ for both retrieval events together and anisomycin decreased the freezing level in

383 both groups, suggesting that $\mathrm{CO}_{2}$ had equal effects on both tone and noise (Fig. s4 J-

384 Q). As another control, saline injection following retrieval and $\mathrm{CO}_{2}$ did not cause

385 memory erasure, indicating that anisomycin was necessary to disrupt the reactivated

386 memory (Fig. s5). Talking together, our data demonstrate that the effects of $\mathrm{CO}_{2}$ are

387 specific to a distinct memory that is activated by a specific CS.

The specific effects of $\mathrm{CO}_{2}$ on memory lability is ASIC-dependent.

390 We have previously found the effects of $\mathrm{CO}_{2}$ on memory retrieval to be ASIC dependent

391 (Du et al., 2017). However, it is still unknown if $\mathrm{CO}_{2}$ application to a specific memory

392 trace affects an ASIC-dependent mechanism. To answer this question, we first

393 performed distinct aversive conditioning in $\mathrm{ASIC1} \mathrm{a}^{---}$mice with three pure tones and

394 white noises on day 1 (Fig. 3A), followed by a pure tone and white noise for retrieval

395 on day 2. 30 minutes post-retrieval, we performed extinctions with pure tones. Five days

396 later, we tested spontaneous recovery and renewals with 4 pure tones and white

397 noises. Similar to the response we saw in WT mice, the freezing level in the pure tone 
398 group of $\mathrm{ASIC}^{-1 /-}$ mice were less than that in the white noise group (spontaneous

399 recovery, 46\% decrease; renewal, 47.5\% decrease) (Fig. 3B-E). When 10\% $\mathrm{CO}_{2}$

400 inhalation was paired with pure tone in retrieval, we found that $\mathrm{CO}_{2}$ did not have

401 additional effects on the memory with the specific CS in ASIC1 $1 a^{--}$mice (spontaneous

402 recovery, 43.4\% decrease; renewal, 45.6\% decrease) (Fig. 3F-I), and there are no

403 statistical significances between the groups with or without $\mathrm{CO}_{2}$ in both spontaneous

404 recovery and renewal (green columns, Fig. 3J, K). We had hypothesized that the

405 effects of $\mathrm{CO}_{2}$ on memory retrieval would be ASIC dependent and our data supported

406 this prediction. We then replaced the extinction procedure with anisomycin, to obliterate

407 the aversive memory (Fig. 4A). The ASIC1a-/- mice were conditioned with pure tones

408 (Fig. 4B) and followed by a single tone as retrieval (Fig. 4C). We then apply anisomycin

409 infusions (Fig. 4D)and test the memory 5 days later (Fig. 4E). Anisomycin dramatically

410 reduced freezing in memory tests that followed, whereas pairing with $\mathrm{CO}_{2}$ in the CS did

411 not cause an additional reduction in the ASIC1 $\mathrm{a}^{-/-}$mice, suggesting an ASIC

412 dependency (Fig. 4E). We also presented two distinct CSs (pure tone and white noise)

413 during conditioning and then retrieval, with or without $10 \% \mathrm{CO}_{2}$, followed by anisomycin

414 infusions in the ASIC1 $\mathrm{a}^{-/-}$mice (Fig. 4F). Anisomycin dramatically reduced freezing in

415 memory tests that followed, whereas pairing with $\mathrm{CO}_{2}$ in either CSs (pure tone or white

416 noise) did not cause an additional reduction in the ASIC1 ${ }^{-/-}$mice, suggesting an ASIC

417 dependency (Fig. 4G-N).

419 To provide evidence that an acute ASIC1a blockage was able to eliminate the effects of $420 \mathrm{CO}_{2}$, we injected 100nM PcTX-1 into the lateral amygdala bilaterally 1 hour before the 421 application of $\mathrm{CO}_{2}$ to the retrieval (Fig. 5A). Our data suggest that compared to the 
422 saline injection group (Fig. 5 B-E), inhibiting ASIC1a by PcTX-1 significantly reduced

423 the $\mathrm{CO}_{2}$ effects on memory retrieval (Fig. 5 F-I), statistical analysis in the spontaneous

424 recovery and renewal groups supported this conclusion (green columns, Fig. 5J, K).

425 This pattern of findings suggests that the effects of $\mathrm{CO}_{2}$ on specific memory traces are 426 ASIC dependent.

Activation of ASICs through $\mathrm{CO}_{2}$ inhalation alters reconsolidation of distinct

memory through alteration of AMPARs.

AMPARs are glutamatergic receptors that have crucial roles in modulating memory retrieval and destabilization (Auber et al., 2013; Chan et al., 2010; Clem and Huganir,

432 2010; Monfils et al., 2009; Quirk et al., 2010). Previous studies suggest that the

433 exchange of $\mathrm{Ca}^{2+}$-impermeable AMPARs (Cl-AMPARs) for $\mathrm{Ca}^{2+}$-permeable AMPARs

434 (CP-AMPARs) occurs after retrieval (Clem and Huganir, 2010; Hong et al., 2013). 10\%

$435 \mathrm{CO}_{2}$ inhalation paired with retrieval induces a stronger current rectification of AMPARs

436 (the signature of CP-AMPARs) than in the retrieval alone group, indicating a greater

437 exchange of AMPARs (Du et al., 2017). Interestingly, no further enhancement was

438 observed in the $\mathrm{ASIC} 1 \mathrm{a}^{-/-}$brain slices, indicating that the effect of $\mathrm{CO}_{2}$ inhalation on

439 AMPAR exchange is ASIC dependent (Du et al., 2017). To further study whether

$440 \mathrm{CO}_{2}$ specifically alters the AMPARs exchange in retrieval, we designed a unique

441 experiment to separate the aversive conditioning and retrieval and measure the

442 rectification of AMPARs (Fig. 6A). To study this, we conditioned the mice with 6 pure

443 tones on day 1 (Fig. 6B, left). On day 2, the mice were divided into 4 groups based on

444 retrieval conditions - the first group received pure tone only; the second group-pure tone 
445 plus $10 \% \mathrm{CO}_{2}$ inhalation; the third group - white noise only; and the fourth group

446 received white noise $+10 \% \mathrm{CO}_{2}$ inhalation (Fig. 6B, right). Ten minutes after retrieval,

447 we dissected brain slices and AMPAR current was recorded in the pyramidal neurons in

448 the lateral amygdala through stimulation of thalamic inputs (Fig. 6A). Rectification, a

449 signature of CP-AMPARs, was compared among all groups. Consistent with earlier

450 reports, pure tone retrieval increased current rectification (Clem and Huganir, 2010;

451 Hong et al., 2013) and $\mathrm{CO}_{2}$ paired with pure tone retrieval caused stronger rectification

452 (Fig. 6C). However, when white noise was presented as the retrieval event, both white

453 noise and white noise plus $\mathrm{CO}_{2}$ failed to cause a significant rectification compared to

454 the pure tone group (Fig. 6C). This data supports our prediction that $\mathrm{CO}_{2}$ was

455 associated with a specific memory trace that was reactivated. To control for the possible

456 effects of the order of presentation of CSs, we switched over the pure tone and white

457 noise in the aversive conditioning and retrieval. Similar results were observed,

458 confirming the effects of $\mathrm{CO}_{2}$ were not artificial (Fig. 6D, E).

460 We then asked whether synaptic strength changed with the application of an unrelated

461 retrieval $\mathrm{CS}$ and $\mathrm{CO}_{2}$. The ratio of AMPAR-EPSCs to NMDAR-EPSCs might represent

462 the strength of the synapse (Rao and Finkbeiner, 2007). Previous studies reported that

463 the AMPAR/ NMDAR-EPSC ratio increased after aversive conditioning whereas

464 retrieval did not potentiate further increase, suggesting that memory retrieval did not

465 alter the synaptic strength (Clem and Huganir, 2010; Hong et al., 2013). Our previous

466 studies also indicated that $\mathrm{CO}_{2}$ inhalation during memory retrieval did not strengthen the

467 synapse in the amygdala (Du et al., 2017). We further tested whether the pairing of $\mathrm{CO}_{2}$ 
468 inhalation with the specific retrieval CS influenced the strength of a synapse. Currents

469 were recorded at $-80 \mathrm{mV}$ for AMPAR-EPSCs and $+60 \mathrm{mV}$ for NMDAR-EPSCs. Our data

470 suggest that retrieval plus $\mathrm{CO}_{2}$ inhalation did not change the AMPAR/ NMDAR-EPSCs

471 ratio (Fig. 6F). Moreover, the characteristics of miniature EPSCs (mEPSCs), including

472 amplitude, frequency, and decay times, were not altered (Fig. 6G). In addition, the

473 pairing of $\mathrm{CO}_{2}$ with another unrelated $\mathrm{CS}$ in retrieval did not change the strength of the

474 synapse (Fig. 6F, G). This data suggests that the effects of $\mathrm{CO}_{2}$ inhalation on specific

475 memory retrieval enhance the destabilization of the synapse without changing synaptic

476 strength.

478 The effects of $\mathrm{CO}_{2}$ inhalation on distinct memory trace.

479 Our previous studies indicate that $\mathrm{CO}_{2}$ enhances memory trace that is associated with

480 aversive conditioning (Du et al., 2017). In this experiment, we examined the mechanism

481 behind the specificity of $\mathrm{CO}_{2}$ effects on memory traces. Using the TetTag-c-fos driven-

482 GFP mouse model, neurons in the amygdala involved in memory trace after aversive

483 conditioning can be labeled with a long-lasting mCherry fluorescent protein and the

484 neuron in the retrieval trace can be labeled with a 2-hour short half-life nuclear-localized

485 EGFP (shEGFP) (Fig. 7A, B) (see the details in Materials and Methods) (Du et al.,

486 2017; Koffman and Du, 2017). The overlapped labeling (yellow) represents the neurons

487 in the same memory trace of aversive and retrieval (Du et al., 2017; Liu et al., 2012;

488 Ramirez et al., 2013). In this experiment, the mice were first conditioned with pure tone,

489 activating the associated neurons that were labeled with mCherry (Fig. 7C). Right

490 after aversive conditioning, the mice were immediately fed with a DOX diet thereby 
491 preventing further mCherry labeling. On day 2, the mice were divided into two groups-

492 one group of mice were presented with a single pure tone to retrieve the memory,

493 another was presented with white noise. A temporary, 2-hour half-life, shEGFP was

494 labeled after the retrieval. Thirty minutes after the retrieval event, we sliced the

495 amygdala and imaged shEGFP- and mCherry-positive cells (Fig. 7D). Compared to

496 pure tone aversive conditioning/pure tone retrieval group (same memory trace of

497 aversive conditioning and retrieval), inhalation of $\mathrm{CO}_{2}$ in the pure tone aversive

498 conditioning/white noise retrieval group did not result in an increase of neurons positive

499 for expression of both mCherry-positive and shEGFP-positive neurons (overlapped

500 labeling, yellow) (Fig. 7E). Control experiments to identify the efficiency of the aversive

501 conditioning on the expression of mCherry on the cells were performed (Fig. 7F). These

502 findings indicate that $\mathrm{CO}_{2}$, when paired with retrieval, only enhances the memory trace

503 that has been reactivated; $\mathrm{CO}_{2}$ paired with unrelated retrieval cue does not affect the

504 original memory trace. These findings suggest a specific effect of $\mathrm{CO}_{2}$ on the memory

505 trace.

507 We then examined the effects of $\mathrm{CO}_{2}$ on dendritic spine morphology after memory

508 retrieval. Spine morphology has been widely indicated in the mechanism of synaptic

509 plasticity (Woolfrey and Srivastava, 2016; Wright et al., 2020; Yang et al., 2009).

510 Dendritic spines are the primary target of neurotransmission input in the central nervous

511 system (Bourne and Harris, 2008), and their density and structure provide the basis for

512 physiological changes in synaptic efficacy that underlie learning and memory (Bailey et

513 al., 2015). Spine formation and plasticity are regulated by many conditions, including 
514 exterior stimulation and behavior (Gipson and Olive, 2017). We hypothesized that $\mathrm{CO}_{2}$

515 inhalation during retrieval alters both structure and plasticity of dendritic spines. The

516 molecular mechanism by which $\mathrm{CO}_{2}$ regulates spine plasticity may explain how $\mathrm{CO}_{2}$

517 converts memory into the labile stage.

519 Using the TetTag-c-fos driven-GFP mouse model, we imaged spine structure and

520 assessed spine density and morphology in overlapping neurons of the amygdala in

521 each group (pure tone aversive conditioning, pure tone retrieval and pure tone aversive

522 conditioning, white noise retrieval) (Fig. 7A, C). Mature spines - most of which display

523 "mushroom-like" morphology - have more stable postsynaptic structures enriched in

524 AMPARs. In contrast, immature spines with a "thin-like" morphology, are unstable

525 postsynaptic structures that have the transitional ability. Immature dendritic spines are

526 thought to be responsible for synaptic plasticity, as they have the potential for

527 strengthening (Berry and Nedivi, 2017). The categories of spines were identified based

528 on the parameters in the previous studies (Fig. 7G) (see the details in the Material and

529 Methods) (Kreple et al., 2014; Wright et al., 2020). The behavior procedure was

530 described in Fig. 7C, the animals underwent aversive conditioning with a tone as a

531 CS and followed by a retrieval on day 2 with tone or noise. We found increased spine

532 numbers after aversive conditioning, indicating that aversive conditioning increases

533 synaptic strength. There was no additional increase in the density of spines in all

534 groups, suggesting that retrieval and $\mathrm{CO}_{2}$ inhalation did not change the synaptic

535 strength (Fig. 7H, lower-right). 
537 We further analyzed spine subtypes as described in the experimental procedures. Thin

538 spines are deemed to represent the immature structure of the synapses (Berry and

539 Nedivi, 2017). We examined the ratio of the number of thin spines to the total number of

540 spines. Interestingly, we found that there were more thin spines in the retrieval group,

541 which might suggest a greater potential for synaptic plasticity in this group (Fig. 7H,

542 lower-middle). Importantly, when the retrieval group (tone) was paired with $\mathrm{CO}_{2}$, we

543 found an additional increase of thin spines compared to the retrieval group alone (Fig.

$5447 \mathrm{H}$, lower-middle). This finding suggests that $\mathrm{CO}_{2}$ paired with retrieval might boost

545 synaptic plasticity compared to memory retrieval alone. Consistently, the mushroom

546 spine numbers decreased in the tone and $\mathrm{CO}_{2}$ paired retrieval groups, suggesting a

547 higher turnover rate after memory retrieval (Fig. 7H, lower-left). However, when the

548 mice were trained with the pure tone and during the retrieval session were presented

549 with white noise (a generated unrelated CS), we found that in the noise group with or

550 without $\mathrm{CO}_{2}$ inhalation, the thin spine number did not increase compared to the pure

551 tone/retrieval group (Fig. 7H, lower-middle). This finding supports the specific effect of

$552 \mathrm{CO}_{2}$ on the memory trace.

\section{DISCUSSION}

555 A newly acquired aversive memory is labile and can be easily disrupted before it is

556 transformed into a long-term stable state (Alberini, 2011). An existing memory, when

557 reactivated, may become labile again during a short post-reactivation period known are

558 reconsolidation window (Schiller et al., 2012). Previous studies using auditory aversive

559 conditioning found that a retrieval event utilizing a single tone CS renders the memory

560 labile during the reconsolidation window (Monfils et al., 2009). During this 
561 reconsolidation window, memory is sensitive to the updating processes that may either

562 enhance or weaken the original memory (Bouton et al., 2020; Du et al., 2017;

563 Fukushima et al., 2014). The reconsolidation window offers an opportunity to determine

564 the mechanisms underlying the lability of an existing memory. We have recently found

565 that when mice inhale $10 \% \mathrm{CO}_{2}$ during retrieval, memory lability increases, and the

566 original memory may be replaced through the update mechanisms (Du et al., 2017).

567 Here, we show that the effects of $\mathrm{CO}_{2}$ on memory are specific to the reactivated

568 conditioned cue (Debiec et al., 2010; Doyere et al., 2007). To address the specificity of

569 the effects of $\mathrm{CO}_{2}$ on reactivated memories, we used a unique behavioral approach with

570 distinct CSs and US, which allowed us the creation of distinct memories. An application

571 of $\mathrm{CO}_{2}$ to the reactivated but not no-reactivated memory enhanced its lability.

572

573 We then asked about the cellular mechanisms through which $\mathrm{CO}_{2}$ enhances the lability

574 of aversive memory. Memory retrieval-induced lability is glutamate receptor-dependent

575 (Rose and Rankin, 2006; Milton, A.L., Everitt, B.J. 2013). We have shown that $\mathrm{CO}_{2}$

576 activates $\mathrm{ASIC1}$ a by decreasing $\mathrm{pH}$ in the brain during retrieval and the activation of

577 ASIC1a increases postsynaptic intracellular calcium and increases AMPAR exchange

578 (Du et al., 2017). Previous research studying the mechanism of retrieval of aversive

579 memories have revealed the rapid and transient exchange from Cl-AMPARs to CP-

580 AMPARs in the lateral amygdala synapses (Clem and Huganir, 2010; Hong et al., 2013)

581 after presenting the CS. In this study, we focused on the specificity of $\mathrm{CO}_{2}$ effects on the

582 exchange of the AMPARs in the lateral amygdala's synapses. We conditioned the mice

583 with a pure tone CS and reactivated the memory with the pure tone with or without

$584 \mathrm{CO}_{2}$ inhalation. Consistent with our earlier findings, we observed that $\mathrm{CO}_{2}$ increases 
AMPAR exchange when it is paired with retrieval. However, when mice were presented with an unrelated $\mathrm{CS}$ during the retrieval stage, $\mathrm{CO}_{2}$ did not alter the AMPAR exchangesuggesting the effects of $\mathrm{CO}_{2}$ on memory trace are memory specific. In addition, synaptic strength (ratio of AMPAR/NMDAR and amplitude of the mEPSCs) was not altered while applying $\mathrm{CO}_{2}$ during retrieval, with or without combining with the memory trace. The exchange of Cl-AMPARs to CP-AMPARs indicates a synaptic plasticity change. Interestingly, $\mathrm{CO}_{2}$ did not increase the total number of spines compared to the

592 retrieval alone group, which suggests the strength of the synapse in a memory trace 593 does not change. In addition, thin spine density significantly increased when retrieval

594 was combined with $\mathrm{CO}_{2}$ inhalation, suggesting that $\mathrm{CO}_{2}$ application changes plasticity.

595 Using this model, we found when an unrelated CS was presented during the retrieval session, no additional increase of immature spine density occurred. This finding

597 indicates no plasticity change in the memory trace. Thus, we can conclude from our 598 findings that the effects of $\mathrm{CO}_{2}$ on memory trace are specific.

Although we have provided evidence that $\mathrm{CO}_{2}$ indeed acts with specificity on a memory

601 trace, this study did not determine how $\mathrm{CO}_{2}$ directly regulates a memory. We cannot

602 exclude the possibility that $\mathrm{CO}_{2}$ might trigger specific effects on memory through other

603 targets. For instance, $\mathrm{CO}_{2}$ inhalation increases cerebral blood flow and arterial blood 604 pressure and might affect brain functions, such as cognition. Although no direct 605 evidence supports the possibility that increased cerebral blood flow and arterial blood 606 pressure affect learning and memory, future studies will have the mechanisms 607 underlying the specificity of $\mathrm{CO}_{2}$ on memory. Future studies will also have to examine 
608 whether $\mathrm{CO}_{2}$ has similar effects on synapses in other brain regions and on other learned

609 behaviors (e.g. appetitive behaviors and drug addictive behaviors).

611 In conclusion, the effects of $\mathrm{CO}_{2}$ on the lability of aversive memory are found to be

612 specific under certain conditions. Our research tests the novel hypothesis that protons

613 are neurotransmitters that activate the postsynaptic proton receptors, ASICs, to

614 manipulate memory updates. This non-invasive, drug-free methodology is innovative,

615 efficacious, and safe for translation to clinical use. As a result, this research may lead to

616 an effective complementary treatment for many mental health-related disorders for

617 which efficient treatments are lacking. We hope this research will lead to new areas of

618 inquiry into $\mathrm{CO}_{2}$-related mechanisms that underlie memory modification and lead to the

619 development of novel therapies for anxiety disorders such as PTSD.

\section{ACKNOWLEDGMENTS}

622 We thank Olivia Miller, Melissa Curtis, Nora Abdul-Aziz, Rida Naqvi, Caitlin Kilmurry,

623 Becca Sturges, Jen Page, Chase Carr, Jordan Jones for their assistance. We thank

624 Drs. Susumu Tonegawa for providing the TRE-mCherry plasmid. J.Du. is supported by

625 the National Institutes of Mental Health (1R01MH113986), the University of Toledo

626 start-up fund, and the University of Tennessee Health Science Center start-up fund.

\section{AUTHOR CONTRIBUTIONS}

629 J.DU., J.D., E.K., C.K. and conceived the project. J.DU., E.K., C.K. and J.D. designed

630 the experiments. E.K., C.K., J.E., S.B., M.H., B.L., performed the behavior experiments. 
631 K.S., performed the spine morphology experiments and data analysis. F.N., and J.DU.

632 performed the patch-clamp experiments and data analysis. E.K., and J.DU., wrote the

633 manuscript. All authors reviewed and edited the manuscript.

634

635 DECLARATION OF INTERESTS: The authors declare no competing financial

636 interests.

637

638

639

640

641

642

643

644

645

646

647

648

649

650

651

652

653 


\section{FIGURE LEGENDS}

655 Figure 1: $\mathrm{CO}_{2}$ inhalation during a selective memory retrieval potentiates the effect

656 of the extinction. (A) Representative schematic of protocol for the aversive

657 conditioning (Av. Cond.), memory retrieval (Ret), extinction (Ext), and memory test-

658 spontaneous recovery (Spon Rec) and renewal. On day1, the mice were subjected to 3

659 pure tones and 3 white noises, paired with 6 foot shocks in context A. One day after, the

660 mice were placed in context B and were subjected to both tone and noise as retrieval

661 events. 30 mins after retrieval, the mice were treated with 2 blocks of extinctions with a

662 pure tone as the CS. On day 7, spontaneous recovery and renewal were tested

663 individually in context B and then context A. 4 pure tones and 4 white noises were

664 presented as CSs during each memory testing. (B-E) Data are presented by the

665 percentage of freezing time during the CSs (tone and noise) in aversive conditioning

666 (B), retrievals (tone and noise) (C), two sections of extinction with tone (D), memory test

667 of spontaneous recovery (Spon Rec) and renewal with tone and noise (E). (F-I) Data

668 are presented by the percentage of freezing time during the CSs (tone and noise) in

669 aversive conditioning (F), retrievals (tone plus $\mathrm{CO}_{2}$ inhalation and noise) (G), two

670 sections of extinction with tone $(\mathbf{H})$, memory test of spontaneous recovery (Spon Rec)

671 and renewal with tone and noise (I). (J-K), comparison data based on spontaneous

672 recovery and renewal respectively from panels $\mathbf{E}$ and $\mathbf{I}$. Data are mean \pm SEM. $\mathrm{n}=10$ -

67312 mice in each group. 'n.s.' indicates not statistically significant. * indicates $p<0.05,{ }^{* *}$

674 indicates $p<0.01,{ }^{* * *}$ indicates $p<0.001,{ }^{* * * *}$ indicates $p<0.0001$, by unpaired Student's t-

675 test and one-way ANOVA with Tukey's post-hoc multiple comparisons.

676 See also Figure S1 and Figure S2. 


\section{Figure 2: $\mathbf{C O}_{2}$ inhalation during memory retrieval potentiates the effect of the} anisomycin. (A) Representative schematic of the protocol for aversive conditioning, memory retrieval, anisomycin injection, and memory test (spontaneous recovery and renewal). On day1, the mice were subjected to 6 pure tones, paired with 6 foot shocks

682 in context A. One day after CS, the mice were placed in context B and were subjected

683 to one single tone as a retrieval event with or without $\mathrm{CO}_{2}$ inhalation. 30 mins after 684 retrieval, the mice were then infused with $62.5 \mu \mathrm{g} / \mu \mathrm{l}$ anisomycin or saline and then

685 returned to their home cage. On day 7 , spontaneous recovery and renewal were tested 686 individually in context B and then context A. 4 pure tones were presented as CSs during 687 each memory testing. (B-E) Data are presented by the percentage of freezing time 688 during the tone presentation in aversive conditioning (B), retrieval (tone) $(\mathbf{C})$, saline or 689 anisomycin infusion in the amygdala (D), spontaneous recovery (Spon Rec), and

690 renewal test with tones (E). (F-I) Data are presented by the percentage of freezing time 691 during the tone presentation in aversive conditioning (F), retrieval (tone) with or without $692 \mathrm{CO}_{2}(\mathbf{G})$, anisomycin infusion $(\mathbf{H})$, spontaneous recovery (Spon Rec), and renewal test 693 with tones (I). Data are mean \pm SEM. $n=8-10$ mice in each group. * indicates $p<0.05$, $694^{* * *}$ indicates $p<0.001$, by unpaired Student's t-test. See also Fig. s3, s4, s5

696 Figure 3: The effect of $\mathrm{CO}_{2}$ inhalation on selective memory retrieval is ASIC1adependent. (A) Representative schematic of protocol for the aversive conditioning, 698 memory retrieval, extinction, and memory test (spontaneous recovery and renewal) in 699 ASIC1 ${ }^{---}$mice. On day1, the mice were subjected to 3 pure tones and 3 white noises, 700 paired with 6 foot shocks in context A. One day later, the mice were placed in context B 701 and subjected to both tone and noise as retrieval events. 30 mins after retrieval, the 
702 mice were treated with 2 blocks of extinctions with a pure tone as the CS. On day 7 ,

703 spontaneous recovery and renewal were tested individually in context B and then

704 context A. 4 pure tones and 4 white noises were presented as CSs during each memory

705 testing. (B-E) Data in ASIC1 ${ }^{-/-}$mice are presented by the percentage of freezing time

706 during the CSs (tone and noise) in aversive conditioning (B), retrievals (tone and noise)

707 (C), two sections of extinction with tone (D), memory test of spontaneous recovery

708 (Spon $\mathrm{Rec}$ ) and renewal with tone and noise (E). (F-I) Data in ASIC1 $1 \mathrm{a}^{-/-}$mice in

709 aversive conditioning (F), retrievals (pure tone plus $10 \% \mathrm{CO}_{2}$ inhalation and white noise)

710 (G), two sections of extinction with pure tone $(\mathbf{H})$, memory test of spontaneous recovery

711 (Spon Rec) and renewal with tone and noise (I). (J-K), comparison data based on

712 spontaneous recovery and renewal respectively from panels $\mathbf{E}$ and I. Data are mean \pm

713 SEM. $\mathrm{n}=12-16$ mice in each group. * indicates $\mathrm{p}<0.05,{ }^{* *}$ indicates $\mathrm{p}<0.01,{ }^{* * *}$

714 indicates $p<0.001$, by unpaired Student's t-test and one-way ANOVA with Tukey's post-

715 hoc multiple comparisons.

\section{Figure 4: The $\mathrm{CO}_{2}$ potentiated anisomycin effects are ASIC1a-dependent.}

718 (A) Representative schematic of protocol for the aversive conditioning, memory

719 retrieval, anisomycin injection, and memory test (spontaneous recovery and renewal).

720 On day 1, the mice were subjected to 6 pure tones, paired with 6 foot shocks in context

721 A. One day after, the mice were placed in context B and subjected to one single tone as

722 a retrieval event with or without $\mathrm{CO}_{2}$ inhalation. 30 mins after retrieval, the mice were

723 infused with $62.5 \mu \mathrm{g} / \mu \mathrm{l}$ anisomycin or saline and then returned to their home cage. On

724 day 7 , spontaneous recovery and renewal were tested individually in context B and then 
725 context A. 4 pure tones were presented as CSs during each memory testing. (B-E) Data

726 in $\mathrm{ASIC1} 1 \mathrm{a}^{-/-}$mice are presented by the percentage of freezing time during the tone

727 presentation in aversive conditioning (B), retrieval (tone) with or without $\mathrm{CO}_{2}(\mathbf{C})$,

728 anisomycin infusion in the amygdala (D), spontaneous recovery (Spon Rec) and

729 renewal test with tones (E). (F) Representative schematic of protocol for the aversive

730 conditioning, memory retrieval, extinction, and memory test (spontaneous recovery and

731 renewal). On day $1, \mathrm{ASIC}^{-/-}$mice were subjected to 3 pure tones and 3 white noises,

732 paired with 6 foot shocks in context A. One day later, the mice were placed in context B

733 and subjected to both tone and noise as retrieval events. 30 mins after retrieval, the

734 mice were infused with anisomycin or saline and returned to their home cage. On day 7,

735 spontaneous recovery and renewal were tested individually in context B and then

736 context A. 4 pure tones and 4 white noises were presented as CSs during each memory

737 testing. (G-J) Data are presented by the percentage of freezing time during the tone

738 presentation in aversive conditioning $(\mathbf{G})$, retrieval (noise plus $\mathrm{CO}_{2}$ inhalation, then tone)

739 (H), anisomycin infusion in the amygdala (I), spontaneous recovery (Spon Rec), and

740 renewal test with tones $(\mathbf{J})$. (K-N) Data are presented by the percentage of freezing time

741 during the tone presentation in aversive conditioning (K), retrieval (tone) with or without

$742 \mathrm{CO}_{2}(\mathrm{~L})$, anisomycin infusion in the amygdala (M), spontaneous recovery (Spon Rec),

743 and renewal test with tones $(\mathbf{N})$. Data are mean \pm SEM. $n=12-16$ mice in each group.

744 'n.s.' indicates not statistically significant by unpaired Student's t-test between groups.

746 Figure 5: Blockage of ASIC1ain the amygdala reduces the $\mathrm{CO}_{2}$ effects on

747 selective memory retrieval. (A) Representative schematic of protocol for the aversive

748 conditioning, PcTX-1 injection, memory retrieval, extinction, and memory test 
749 (spontaneous recovery and renewal). On day1, the mice were subjected to 3 pure tones

750 and 3 white noises, paired with 6 foot shocks in context A. One day later, the mice were

751 injected with $100 \mathrm{nM}$ PcTX-1 or saline, then the mice were placed in context B and

752 subjected to both tone and noise as retrieval events with or without $\mathrm{CO}_{2} .30$ mins after

753 retrieval, the mice were treated with 2 blocks of extinctions with a pure tone as the CS.

754 On day 7 , spontaneous recovery and renewal were tested individually in context B and

755 then context A. 4 pure tones and 4 white noises were presented as CSs during each

756 memory testing. (B-E) Data are presented by the percentage of freezing time during the

757 CSs (tone and noise) in aversive conditioning (B), retrievals (tone plus $\mathrm{CO}_{2}$ inhalation

758 and noise) after saline injection in the amygdala (C), two sections of extinction with tone

759 (D), memory test of spontaneous recovery (Spon Rec) and renewal with tone and noise

760 (E). (F-I) Data are presented by the percentage of freezing time during the CSs (tone

761 and noise) in aversive conditioning (F), retrievals (tone plus $\mathrm{CO}_{2}$ inhalation and noise)

762 after PcTx-1 injection in the amygdala $(\mathbf{G})$, two sections of extinction with tone $(\mathbf{H})$,

763 memory test of spontaneous recovery (Spon Rec) and renewal with tone and noise (I).

$764(\mathrm{~J}-\mathrm{K})$, comparison data based on spontaneous recovery and renewal respectively from

765 panels $\mathbf{E}$ and I. Data are mean \pm SEM. $\mathrm{n}=12$ mice in each group. ' $\mathrm{n}$.s.' indicates not

766 statistically significant. * indicates $p<0.05,{ }^{* *}$ indicates $p<0.01,{ }^{* * *}$ indicates $p<0.001,{ }^{* * * *}$

767 indicates $p<0.0001$, by unpaired Student's t-test and one-way ANOVA with Tukey's

768 post-hoc multiple comparisons.

770 Figure 6: $\mathrm{CO}_{2}$ inhalation during a selective memory retrieval enhances the

771 retrieval dependent AMPAR current rectification. (A) Representative schematic of 
772 the protocol. On day 1, the animal underwent 6 CSs (tones or noses), paired with 6-foot

773 shocks in context A. On day 2, the mice were divided into 4 groups for the retrieval-pure

774 tone only; pure tone plus $10 \% \mathrm{CO}_{2}$ inhalation; white noise only; white noise+10\% $\mathrm{CO}_{2}$

775 inhalation. Ten minutes after retrieval, the brain slices were dissected and AMPAR

776 current was recorded in the pyramidal neurons in the lateral amygdala through

777 stimulation of thalamic input. (B-E) Mice underwent 6 pure tones (B) or 6 noises (D) in

778 aversive conditioning, Data are presented by the percentage of freezing time during the

779 tone presentation in aversive conditioning, retrieval (noise plus $\mathrm{CO}_{2}$ inhalation, then

780 tone). (C, E) Left, AMPARs current-voltage relationships in the recorded neurons. Insets

781 show an example of the AMPAR-EPSCs in $-80 \mathrm{mV}$ and $+60 \mathrm{mV}$. Right, AMPAR

782 rectification index (I-80 $\mathrm{mV} / \mathrm{I}+60 \mathrm{mV})$. Data are mean \pm SEM. $\mathrm{n}=20-26$ for each group.

783 (F) Left, examples of EPSC recordings of AMPAR-EPSCs (-80mV) and NMDAR-

784 EPSCs (+60mV). Right, AMPAR/NMDAR EPSC ratios. Current amplitudes were

785 measured 70 ms after onset. $n=20-26$ for each group. (G) Miniature EPSCs recordings

786 from the neurons after retrieval. Upper, representative mEPSC traces from different

787 groups. Lower, cumulative distributions of mEPSC amplitudes, inter-event intervals, and

788 decay-times. $n=25-40$ for each group. Data are mean \pm SEM. 'n.s.' indicates not

789 statistically significant. * indicates $p<0.05,{ }^{* *}$ indicates $p<0.01$, by ANOVA with Tukey's

790 post hoc multiple comparisons.

792 Figure 7: $\mathbf{C O}_{2}$ inhalation during a selective memory retrieval enhances the

793 retrieval-related memory trace. (A) Schematic showing the c-Fos-tTA-GFP mouse

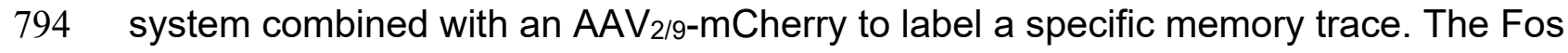


795 promoter in transgenic mice was activated by activities, followed by a transient, 2-hour

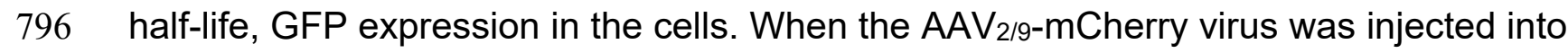

797 the brain, the activation of c-FOS also induces the expression of mCherry. When the

798 mice were fed with DOX, the mCherry expression was interrupted. (B) an example

799 image showing the efficiency of the expression of GFP and mCherry in the amygdala.

800 (C) The procedure of aversive conditioning, memory retrieval, and memory trace

801 labeling using the system in A. Mice was fed with DOX for at least one week, followed

802 by an injection of $\mathrm{AAV}_{2 / 9}-\mathrm{mCherry}$ in the amygdala. Two weeks later, DOX was removed

803 and mice were subjected to aversive conditioning with a pure tone as the CS. DOX has

804 added back again immediately after aversive conditioning. 24 hours later, the mice

805 underwent retrieval with pure tone or noise. The brain slices were collected 30 mins

806 after retrieval and then were stained for microscopy. (D) Left, representative images of

807 the neurons labeled by mCherry, GFP, and DAPI; Right, the enlarged area from the

808 "merge" image showing the overlapping expression of mCherry and GFP neurons. The

809 overlapping neurons indicate their "consanguinity" in the same memory trace. (E)

810 Summarized data are the percentage of the overlapping expression of mCherry and

811 GFP neurons in different behavior groups. All mice underwent aversive conditioning

812 with a tone as the CS. 24 hours later, the mice were separated into 4 groups. Tone

813 group: retrieval CS by tone; Tone $+\mathrm{CO}_{2}$ group: retrieval CS by tone along with $\mathrm{CO}_{2}$

814 inhalation; Noise group: retrieval CS by noise; Noise $+\mathrm{CO}_{2}$ group: retrieval CS by noise

815 along with $\mathrm{CO}_{2}$ inhalation. (F) Control experiment showing the expression of mCherry

816 with or without DOX as well as with or without aversive conditioning. (G) Left, a

817 representative image showing the spine morphology in the mCherry and GFP 
818 colocalized neurons. The mature spines were categorified as "mushroom" spines and

819 the immature spines were categorified as "thin" spines; Right, an enlarged image

820 showing the details of mushroom and thin spines. (H) Upper, representative images of

821 the spine structures in different animal groups showed in E; Lower, summarized data of

822 the spine densities of mushroom spines, thin spines, and total spines in the different

823 groups. Data are mean \pm SEM. $n=8-12$ mice for each group. 'n.s.' indicates not

824 statistically significant. * indicates $p<0.05,{ }^{* * *}$ indicates $p<0.001,{ }^{* * * *}$ indicates

$825 \mathrm{p}<0.0001$, by unpaired Student's t-test and one-way ANOVA with Tukey's post-hoc

826 multiple comparisons.

828 Figure s1: White noise was an appropriate successful CS to involve use in

829 studying the effects of $\mathrm{CO}_{2}$ effects on aversive conditioning and retrieval.

830 (A) Representative schematic of protocol for the aversive conditioning (pure tone and

831 white noise), memory retrieval (pure tone and white noise), extinction (white noise), and

832 memory test (spontaneous recovery and renewal). (B-E) Data are presented by the

833 percentage of freezing time during the CSs (tone and noise) in aversive conditioning

834 (B), retrievals (tone and noise) (C), two sections of extinction with white noise (D),

835 memory test of spontaneous recovery (Spon Rec) and renewal with tone and noise (E).

836 (F-I) Data are presented by the percentage of freezing time during the CSs (tone and

837 noise) in aversive conditioning (F), retrievals (pure tone and white noise plus $\mathrm{CO}_{2}$

838 inhalation) (G), two sections of extinction with white noise $(\mathbf{H})$, memory test of

839 spontaneous recovery (Spon Rec) and renewal with tone and noise (I). (J-K),

840 comparison data based on spontaneous recovery and renewal respectively from panels 
$841 \quad \mathbf{E}$ and I. Data are mean \pm SEM. $\mathrm{n}=12$ mice in each group. 'n.s.' indicates not

842 statistically significant. * indicates $p<0.05,{ }^{* *}$ indicates $p<0.01,{ }^{* * *}$ indicates $p<0.001,{ }^{* * *}$

843 indicates $p<0.0001$, by unpaired Student's t-test and one-way ANOVA with Tukey's

844 post-hoc multiple comparisons.

Figure s2: $\mathrm{CO}_{2}$ inhalation does not boost extinction when pairs with an unrelated

847 CS during memory retrieval. (A) Representative schematic of protocol for the aversive

848 conditioning (pure tone and white noise), memory retrieval (pure tone and white noise),

849 extinction (white noise), and memory test (spontaneous recovery and renewal). (B-

850 E) Data are presented by the percentage of freezing time during the CSs (tone and

851 noise) in aversive conditioning (B), retrievals (pure tone and white noise plus $\mathrm{CO}_{2}$

852 inhalation) (C), two sections of extinction with pure tone (D), memory test of

853 spontaneous recovery (Spon Rec) and renewal with tone and noise, Blue arrow and \%

854 indicated the difference (decreases) between the tone and noise groups (E). (F-I) Data

855 are presented by the percentage of freezing time during the CSs (tone and noise) in

856 aversive conditioning (F), retrievals (pure tone plus $\mathrm{CO}_{2}$ inhalation and white noise) (G),

857 two sections of extinction with white noise $(\mathbf{H})$, memory test of spontaneous recovery

858 (Spon Rec) and renewal with tone and noise, Blue arrow and \% indicated the difference

859 (decreases) between the tone and noise groups. (I). Data are mean \pm SEM. $\mathrm{n}=12$ mice

860 in each group. * indicates $p<0.05$ by unpaired Student's t-test.

862 Figure s3: Anisomycin disrupts the memory within the reconsolidation window.

863 (A) Representative schematic of protocol for the aversive conditioning (pure tone), with

864 or without memory retrieval (pure tone), anisomycin infusion, and memory test 
865 (spontaneous recovery and renewal). (B-E) Data are presented by the percentage of

866 freezing time during the tone presentation in aversive conditioning (B), with or

867 without retrieval (tone) (C), anisomycin infusion in the amygdala (D), spontaneous

868 recovery (Spon $\mathrm{Rec}$ ), and renewal test with tones (E). (F-I) Data are presented by the

869 percentage of freezing time during the CSs (tone and noise) in aversive conditioning

870 (F), retrievals (pure tone and white noise) $(\mathbf{G})$, anisomycin injection in the amygdala $(\mathbf{H})$,

871 memory test of spontaneous recovery and renewal with tone and noise (I). (J-M) Data

872 are presented by the percentage of freezing time during the CSs (tone and noise) in

873 aversive conditioning $(\mathbf{J})$, retrievals (pure tone plus $\mathrm{CO}_{2}$ inhalation and white noise) $(\mathbf{K})$,

874 saline injection in the amygdala (L), memory test of spontaneous recovery and renewal

875 with tone and noise, (M). Data are mean \pm SEM. $n=11-12$ mice in each group. 'n.s.'

876 indicates not statistically significant. * indicates $p<0.05$ by unpaired Student's t-test.

878 Figure s4: $\mathbf{C O}_{2}$ enhances memory retrieval and potentiates the effects of

879 anisomycin on the reconsolidation. (A) Representative schematic of protocol for the

880 aversive conditioning (pure tone and white noise), memory retrieval (pure tone and

881 white noise) with or without $\mathrm{CO}_{2}$ inhalation, anisomycin infusion, and memory test

882 (spontaneous recovery and renewal). (B-E) Data are presented by the percentage of

883 freezing time during the CSs (tone and noise) presentation in aversive conditioning (B),

884 retrieval (tone plus $\mathrm{CO}_{2}$ and noise) (C), anisomycin infusion in the amygdala (D),

885 spontaneous recovery (Spon $\mathrm{Rec}$ ) and renewal test with tone and noise (E). (F-I) Data

886 are presented by the percentage of freezing time during the CSs (tone and noise) in

887 aversive conditioning $(\mathbf{F})$, retrieval (noise plus $\mathrm{CO}_{2}$ and tone) $(\mathbf{G})$, anisomycin infusion in

888 the amygdala $(\mathbf{H})$, memory test of spontaneous recovery (Spon Rec) and renewal with 
889 tone and noise (I). (J-M) Data are presented by the percentage of freezing time during

890 the CSs (tone and noise) in aversive conditioning (J), retrievals (tone and noise) plus

$891 \mathrm{CO}_{2}(\mathbf{K})$, saline infusion in the amygdala (L), memory test of spontaneous recovery

892 (Spon Rec) and renewal with tone and noise (M). (N-Q) Data are presented by the

893 percentage of freezing time during the CSs (tone and noise) in aversive conditioning

894 (N), retrievals (tone and noise) plus $\mathrm{CO}_{2}(\mathbf{O})$, anisomycin infusion in the amygdala $(\mathbf{P})$,

895 memory test of spontaneous recovery (Spon Rec) and renewal with tone and noise (Q).

896 Data are mean \pm SEM. $n=12$ mice in each group. 'n.s.' indicates not statistically

897 significant. * indicate $p<0.05$ by unpaired Student's t-test.

Figure s5: $\mathrm{CO}_{2}$ does not affect memory reconsolidation without anisomycin.

900 (A) Representative schematic of protocol for the aversive conditioning (pure tone and

901 white noise), memory retrieval (pure tone and white noise) with or without $\mathrm{CO}_{2}$

902 inhalation, saline infusion, and memory test (spontaneous recovery and renewal). (B-

903 E) Data are presented by the percentage of freezing time during the CSs (tone and

904 noise) presentation in aversive conditioning (B), retrieval (tone plus $\mathrm{CO}_{2}$ and noise) (C),

905 saline infusion in the amygdala (D), spontaneous recovery (Spon Rec) and renewal test

906 with tone and noise (E). (F-I) Data are presented by the percentage of freezing time

907 during the CSs (tone and noise) in aversive conditioning (F), retrieval (noise plus $\mathrm{CO}_{2}$

908 and tone) $(\mathbf{G})$, saline infusion in the amygdala $(\mathbf{H})$, memory test of spontaneous

909 recovery (Spon Rec) and renewal with tone and noise (I). Data are mean \pm SEM. $n=12$

910 mice in each group. 'n.s.' indicates not statistically significant by unpaired Student's t-

911 test.

912 


\section{REFERENCES}

914 Agren, T., Engman, J., Frick, A., Bjorkstrand, J., Larsson, E.M., Furmark, T., and

915 Fredrikson, M. (2012). Disruption of reconsolidation erases a fear memory trace in the

916 human amygdala. Science 337, 1550-1552.

Alberini, C.M. (2011). The role of reconsolidation and the dynamic process of long-term memory formation and storage. Front Behav Neurosci 5, 12.

Auber, A., Tedesco, V., Jones, C.E., Monfils, M.H., and Chiamulera, C. (2013). Postretrieval extinction as reconsolidation interference: methodological issues or boundary conditions? Psychopharmacology (Berl) 226, 631-647.

Bailey, C.H., Kandel, E.R., and Harris, K.M. (2015). Structural Components of Synaptic Plasticity and Memory Consolidation. Cold Spring Harb Perspect Biol 7, a021758.

Ben-Shahar, Y. (2011). Sensory functions for degenerin/epithelial sodium channels (DEG/ENaC). Adv Genet 76, 1-26.

Berry, K.P., and Nedivi, E. (2017). Spine Dynamics: Are They All the Same? Neuron 96, 43-55.

Bjorkstrand, J., Agren, T., Ahs, F., Frick, A., Larsson, E.M., Hjorth, O., Furmark, T., and Fredrikson, M. (2016). Disrupting Reconsolidation Attenuates Long-Term Fear Memory in the Human Amygdala and Facilitates Approach Behavior. Curr Biol 26, 2690-2695.

Bourne, J.N., and Harris, K.M. (2008). Balancing structure and function at hippocampal dendritic spines. Annu Rev Neurosci 31, 47-67.

Bouton, M.E., Maren, S., and McNally, G.P. (2020). Behavioral and Neurobiological Mechanisms of Pavlovian and Instrumental Extinction Learning. Physiol Rev.

Chan, W.Y., Leung, H.T., Westbrook, R.F., and McNally, G.P. (2010). Effects of recent exposure to a conditioned stimulus on extinction of Pavlovian fear conditioning. Learn Mem 17, 512-521.

Chiang, P.H., Chien, T.C., Chen, C.C., Yanagawa, Y., and Lien, C.C. (2015). ASICdependent LTP at multiple glutamatergic synapses in amygdala network is required for fear memory. Sci Rep 5, 10143.

Chu, X.P., and Xiong, Z.G. (2012). Physiological and pathological functions of acidsensing ion channels in the central nervous system. Curr Drug Targets 13, 263-271.

Clem, R.L., and Huganir, R.L. (2010). Calcium-permeable AMPA receptor dynamics mediate fear memory erasure. Science 330, 1108-1112. 
957 Cohen, J.L., Jackson, N.L., Ballestas, M.E., Webb, W.M., Lubin, F.D., and Clinton, S.M. 958 (2017). Amygdalar expression of the microRNA miR-101a and its target Ezh2 contribute 959 to rodent anxiety-like behaviour. Eur J Neurosci 46, 2241-2252.

Coryell, M.W., Ziemann, A.E., Westmoreland, P.J., Haenfler, J.M., Kurjakovic, Z., Zha, X.M., Price, M., Schnizler, M.K., and Wemmie, J.A. (2007). Targeting ASIC1a reduces innate fear and alters neuronal activity in the fear circuit. Biol Psychiatry 62, 1140-1148.

Costanzi, M., Cannas, S., Saraulli, D., Rossi-Arnaud, C., and Cestari, V. (2011). Extinction after retrieval: effects on the associative and nonassociative components of remote contextual fear memory. Learn Mem 18, 508-518.

Das, A.T., Tenenbaum, L., and Berkhout, B. (2016). Tet-On Systems For Doxycycline-

Debiec, J., Diaz-Mataix, L., Bush, D.E., Doyere, V., and Ledoux, J.E. (2010). The amygdala encodes specific sensory features of an aversive reinforcer. Nat Neurosci 13, 536-537.

Doyere, V., Debiec, J., Monfils, M.H., Schafe, G.E., and LeDoux, J.E. (2007). Synapsespecific reconsolidation of distinct fear memories in the lateral amygdala. Nat Neurosci 10, 414-416.

Du, J., Price, M.P., Taugher, R.J., Grigsby, D., Ash, J.J., Stark, A.C., Hossain Saad, M.Z., Singh, K., Mandal, J., Wemmie, J.A., et al. (2017). Transient acidosis while retrieving a fear-related memory enhances its lability. Elife 6.

Du, J., Reznikov, L.R., Price, M.P., Zha, X.M., Lu, Y., Moninger, T.O., Wemmie, J.A., and Welsh, M.J. (2014). Protons are a neurotransmitter that regulates synaptic plasticity in the lateral amygdala. Proc Natl Acad Sci U S A 111, 8961-8966. (2005). Adenosine and ATP link PCO2 to cortical excitability via pH. Neuron 48, 10111023.

1000

Duvarci, S., and Nader, K. (2004). Characterization of fear memory reconsolidation. J Neurosci 24, 9269-9275.

Farley, S.J., Albazboz, H., De Corte, B.J., Radley, J.J., and Freeman, J.H. (2018). Amygdala central nucleus modulation of cerebellar learning with a visual conditioned stimulus. Neurobiol Learn Mem 150, 84-92.

Fukushima, H., Zhang, Y., Archbold, G., Ishikawa, R., Nader, K., and Kida, S. (2014). Enhancement of fear memory by retrieval through reconsolidation. Elife 3, e02736. 
Gao, J., Duan, B., Wang, D.G., Deng, X.H., Zhang, G.Y., Xu, L., and Xu, T.L. (2005). Coupling between NMDA receptor and acid-sensing ion channel contributes to ischemic neuronal death. Neuron 48, 635-646.

Gipson, C.D., and Olive, M.F. (2017). Structural and functional plasticity of dendritic spines - root or result of behavior? Genes Brain Behav 16, 101-117. protons act as neurotransmitters at vestibular hair cell-calyx afferent synapses. Proc Natl Acad Sci U S A 111, 5421-5426. Choi, S. (2013). AMPA receptor exchange underlies transient memory destabilization on retrieval. Proc Natl Acad Sci U S A 110, 8218-8223.

Huang, F., Zou, G., Li, C., Meng, H., Liu, X., and Yang, Z. (2020). A novelty-retrievalextinction paradigm leads to persistent attenuation of remote fear memories. Sci Rep $10,3319$.

Ishii, D., Matsuzawa, D., Matsuda, S., Tomizawa, H., Sutoh, C., and Shimizu, E. (2012). No erasure effect of retrieval-extinction trial on fear memory in the hippocampusindependent and dependent paradigms. Neurosci Lett 523, 76-81.

\section{Jarome, T.J., Ferrara, N.C., Kwapis, J.L., and Helmstetter, F.J. (2015). Contextual} Information Drives the Reconsolidation-Dependent Updating of Retrieved Fear Memories. Neuropsychopharmacology 40, 3044-3052.

\section{Kawasaki, H., Eguchi, S., Miyashita, S., Chan, S., Hirai, K., Hobara, N., Yokomizo, A.,} Fujiwara, H., Zamami, Y., Koyama, T., et al. (2009). Proton acts as a neurotransmitter for nicotine-induced adrenergic and calcitonin gene-related peptide-containing nervemediated vasodilation in the rat mesenteric artery. J Pharmacol Exp Ther 330, 745-755.

Kida, S. (2020). Function and mechanisms of memory destabilization and reconsolidation after retrieval. Proc Jpn Acad Ser B Phys Biol Sci 96, 95-106.

Klucken, T., Kruse, O., Schweckendiek, J., Kuepper, Y., Mueller, E.M., Hennig, J., and Stark, R. (2016). No evidence for blocking the return of fear by disrupting reconsolidation prior to extinction learning. Cortex 79, 112-122. 
1046 Koffman, E.E., and Du, J. (2017). Labeling Aversive Memory Trace in Mouse Using a Doxycycline-inducible Expression System. Bio-protocol 7, e2578.

Kreple, C.J., Lu, Y., Taugher, R.J., Schwager-Gutman, A.L., Du, J., Stump, M., Wang, Y., Ghobbeh, A., Fan, R., Cosme, C.V., et al. (2014). Acid-sensing ion channels contribute to synaptic transmission and inhibit cocaine-evoked plasticity. Nat Neurosci 17, 1083-1091.

1053

1054

Lee, J.L. (2009). Reconsolidation: maintaining memory relevance. Trends Neurosci 32,

1055 413-420.

1056

1057

Liu, J., Zhao, L., Xue, Y., Shi, J., Suo, L., Luo, Y., Chai, B., Yang, C., Fang, Q., Zhang,

1058

1059 Y., et al. (2014). An unconditioned stimulus retrieval extinction procedure to prevent the return of fear memory. Biol Psychiatry 76, 895-901.

1060

1061

Liu, M.G., Li, H.S., Li, W.G., Wu, Y.J., Deng, S.N., Huang, C., Maximyuk, O., Sukach,

1062 V., Krishtal, O., Zhu, M.X., et al. (2016). Acid-sensing ion channel 1a contributes to

1063

1064 hippocampal LTP inducibility through multiple mechanisms. Sci Rep 6, 23350.

1065

Liu, X., Ramirez, S., Pang, P.T., Puryear, C.B., Govindarajan, A., Deisseroth, K., and Tonegawa, S. (2012). Optogenetic stimulation of a hippocampal engram activates fear memory recall. Nature 484, 381-385.

1068

1069

Mactutus, C.F., Riccio, D.C., and Ferek, J.M. (1979). Retrograde amnesia for old

1070 (reactivated) memory: some anomalous characteristics. Science 204, 1319-1320.

Mango, D., and Nisticò, R. (2019). Acid-Sensing lon Channel 1a Is Involved in N-Methyl D-Aspartate Receptor-Dependent Long-Term Depression in the Hippocampus. Front Pharmacol 10, 555.

Monfils, M.H., Cowansage, K.K., Klann, E., and LeDoux, J.E. (2009). Extinctionreconsolidation boundaries: key to persistent attenuation of fear memories. Science $324,951-955$.

Nader, K., Schafe, G.E., and Le Doux, J.E. (2000). Fear memories require protein synthesis in the amygdala for reconsolidation after retrieval. Nature 406, 722-726.

1084

Ortega-Ramírez, A., Vega, R., and Soto, E. (2017). Acid-Sensing lon Channels as Inflamm 2017, 3728096.

1088 Quirk, G.J., Paré, D., Richardson, R., Herry, C., Monfils, M.H., Schiller, D., and Vicentic, 1089 
1090 Ramirez, S., Liu, X., Lin, P.A., Suh, J., Pignatelli, M., Redondo, R.L., Ryan, T.J., and

1091 Tonegawa, S. (2013). Creating a false memory in the hippocampus. Science 341, 387-

1092391.

1093

1094

Rao, V.R., and Finkbeiner, S. (2007). NMDA and AMPA receptors: old channels, new

1095

1096

1097

1098

1099

tricks. Trends Neurosci 30, 284-291.

Rose, J.K., and Rankin, C.H. (2006). Blocking memory reconsolidation reverses memory-associated changes in glutamate receptor expression. J Neurosci 26, 1158211587.

Sara, S.J. (2000). Retrieval and reconsolidation: toward a neurobiology of remembering. Learn Mem 7, 73-84.

Sara, S.J., and Hars, B. (2006). In memory of consolidation. Learn Mem 13, 515-521. Schiller, D., Monfils, M.H., Raio, C.M., Johnson, D.C., Ledoux, J.E., and Phelps, E.A. (2010). Preventing the return of fear in humans using reconsolidation update mechanisms. Nature 463, 49-53.

Schiller, D., Raio, C.M., and Phelps, E.A. (2012). Extinction training during the reconsolidation window prevents recovery of fear. J Vis Exp, e3893.

Soto, E., Ortega-Ramírez, A., and Vega, R. (2018). Protons as Messengers of

Taugher, R.J., Lu, Y., Fan, R., Ghobbeh, A., Kreple, C.J., Faraci, F.M., and Wemmie, J.A. (2017). ASIC1A in neurons is critical for fear-related behaviors. Genes Brain Behav $16,745-755$.

Tronson, N.C., and Taylor, J.R. (2007). Molecular mechanisms of memory reconsolidation. Nat Rev Neurosci 8, 262-275.

Waldmann, R., Champigny, G., Bassilana, F., Heurteaux, C., and Lazdunski, M. (1997). A proton-gated cation channel involved in acid-sensing. Nature 386, 173-177.

Waldmann, R., and Lazdunski, M. (1998). H(+)-gated cation channels: neuronal acid sensors in the NaC/DEG family of ion channels. Curr Opin Neurobiol 8, 418-424.

Wang, Q., Wang, Q., Song, X.L., Jiang, Q., Wu, Y.J., Li, Y., Yuan, T.F., Zhang, S., Xu, N.J., Zhu, M.X., et al. (2018). Fear extinction requires ASIC1a-dependent regulation of hippocampal-prefrontal correlates. Sci Adv 4, eaau3075.

Wemmie, J.A., Askwith, C.C., Lamani, E., Cassell, M.D., Freeman, J.H., Jr., and Welsh, density and contributes to fear conditioning. J Neurosci 23, 5496-5502. 
1135 Wemmie, J.A., Chen, J., Askwith, C.C., Hruska-Hageman, A.M., Price, M.P., Nolan, 1136 B.C., Yoder, P.G., Lamani, E., Hoshi, T., Freeman, J.H., Jr., et al. (2002). The acidactivated ion channel ASIC contributes to synaptic plasticity, learning, and memory. Neuron 34, 463-477.

Wemmie, J.A., Coryell, M.W., Askwith, C.C., Lamani, E., Leonard, A.S., Sigmund, C.D., and Welsh, M.J. (2004). Overexpression of acid-sensing ion channel 1a in transgenic mice increases acquired fear-related behavior. Proc Natl Acad Sci U S A 101, 36213626.

Wemmie, J.A., Price, M.P., and Welsh, M.J. (2006). Acid-sensing ion channels: advances, questions and therapeutic opportunities. Trends Neurosci 29, 578-586.

Wemmie, J.A., Taugher, R.J., and Kreple, C.J. (2013). Acid-sensing ion channels in pain and disease. Nat Rev Neurosci 14, 461-471.

Woolfrey, K.M., and Srivastava, D.P. (2016). Control of Dendritic Spine Morphological and Functional Plasticity by Small GTPases. Neural Plast 2016, 3025948.

Wright, W.J., Graziane, N.M., Neumann, P.A., Hamilton, P.J., Cates, H.M., Fuerst, L., Spenceley, A., MacKinnon-Booth, N., lyer, K., Huang, Y.H., et al. (2020). Silent synapses dictate cocaine memory destabilization and reconsolidation. Nat Neurosci 23, 32-46.

Wu, C.T., Haggerty, D., Kemere, C., and Ji, D. (2017). Hippocampal awake replay in spinal cord. J Biol Chem 279, 43716-43724.

Yang, G., Pan, F., and Gan, W.B. (2009). Stably maintained dendritic spines are associated with lifelong memories. Nature 462, 920-924. hypercapnia and other disturbances in the acid-base-balance on panic disorder. Hillside J Clin Psychiatry 11, 185-197. Lynch, C.M., Faraci, F.M., Howard, M.A., 3rd, Welsh, M.J., et al. (2009). The amygdala is a chemosensor that detects carbon dioxide and acidosis to elicit fear behavior. Cell 
A Av. Cond.

(Tone, noise) Ret, $\pm \mathrm{CO}_{2} \quad$ Extinction blocks (Tone) Memory tests (Tone, noise)
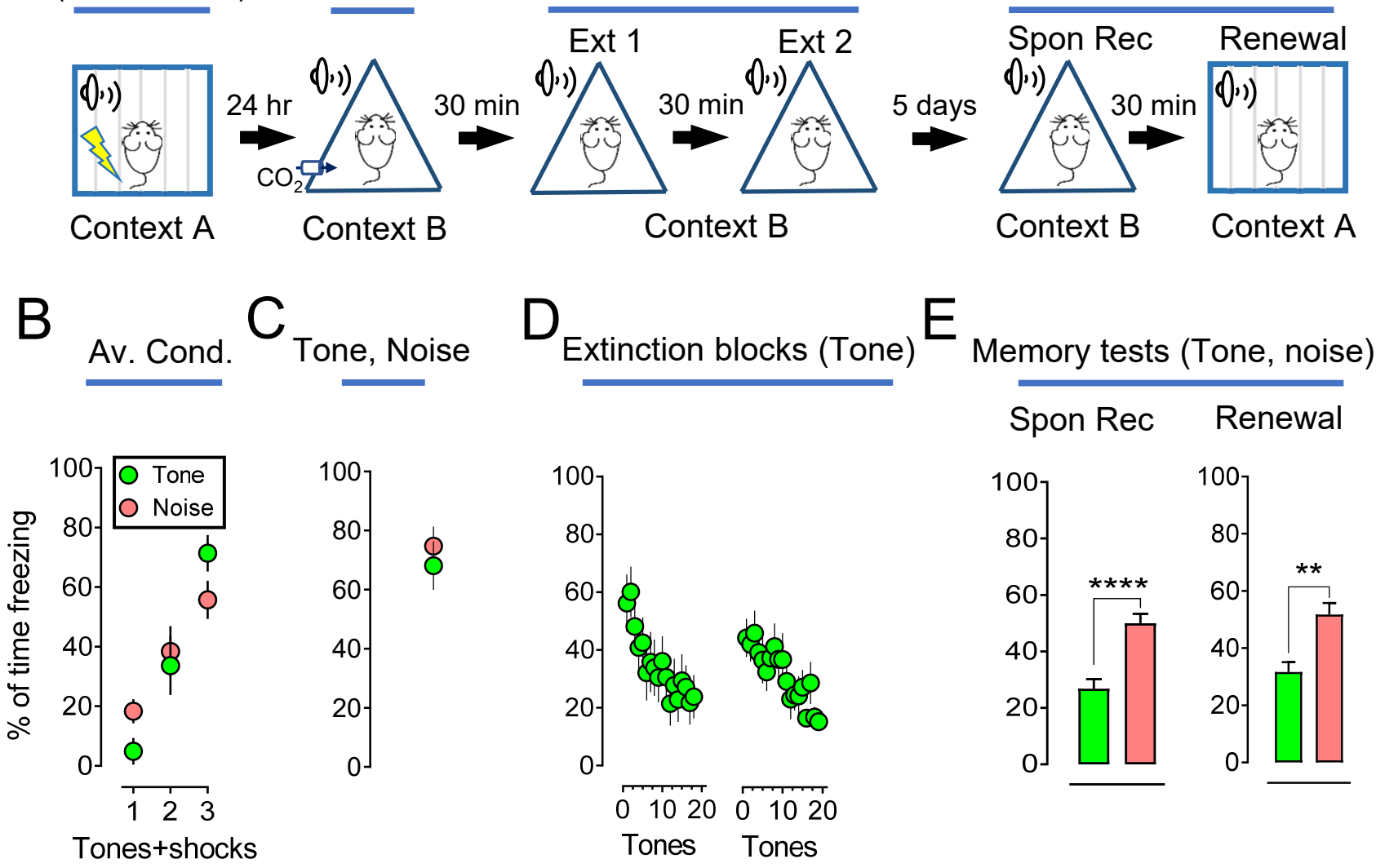

$\mathrm{F}$

$\mathrm{F} \quad \mathrm{G}_{\left(\mathrm{Tone}+\mathrm{CO}_{2}\right),} \mathrm{H}$

Av. Cond.

Noise

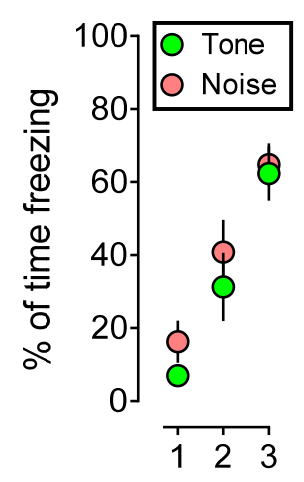

Tones+shocks
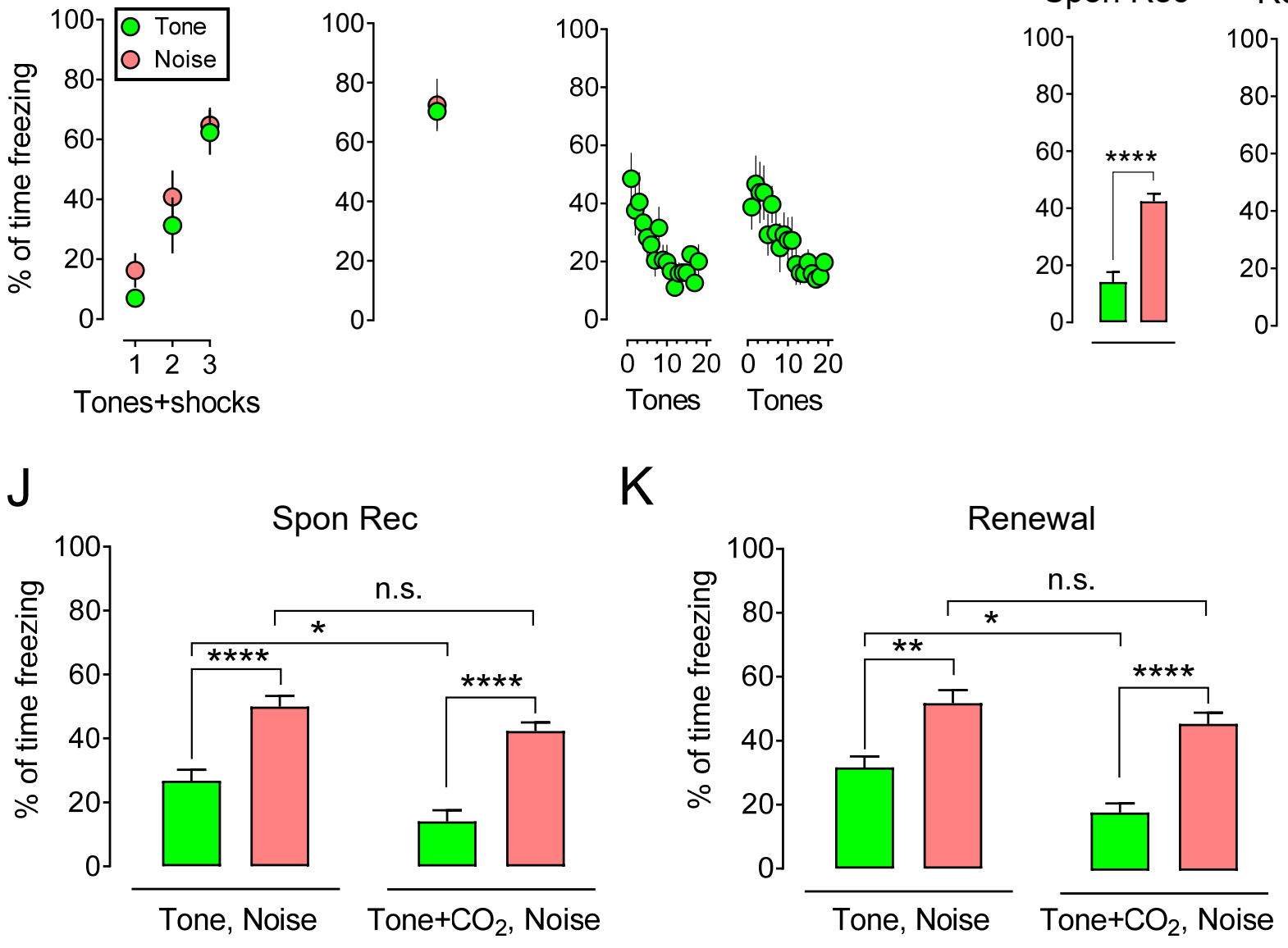

Memory tests (Tone, noise)
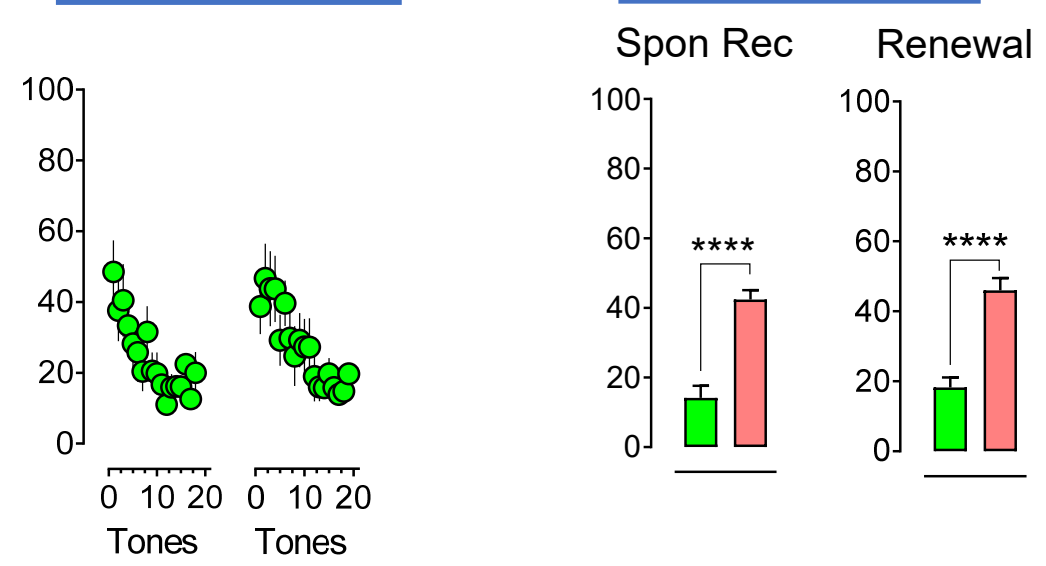

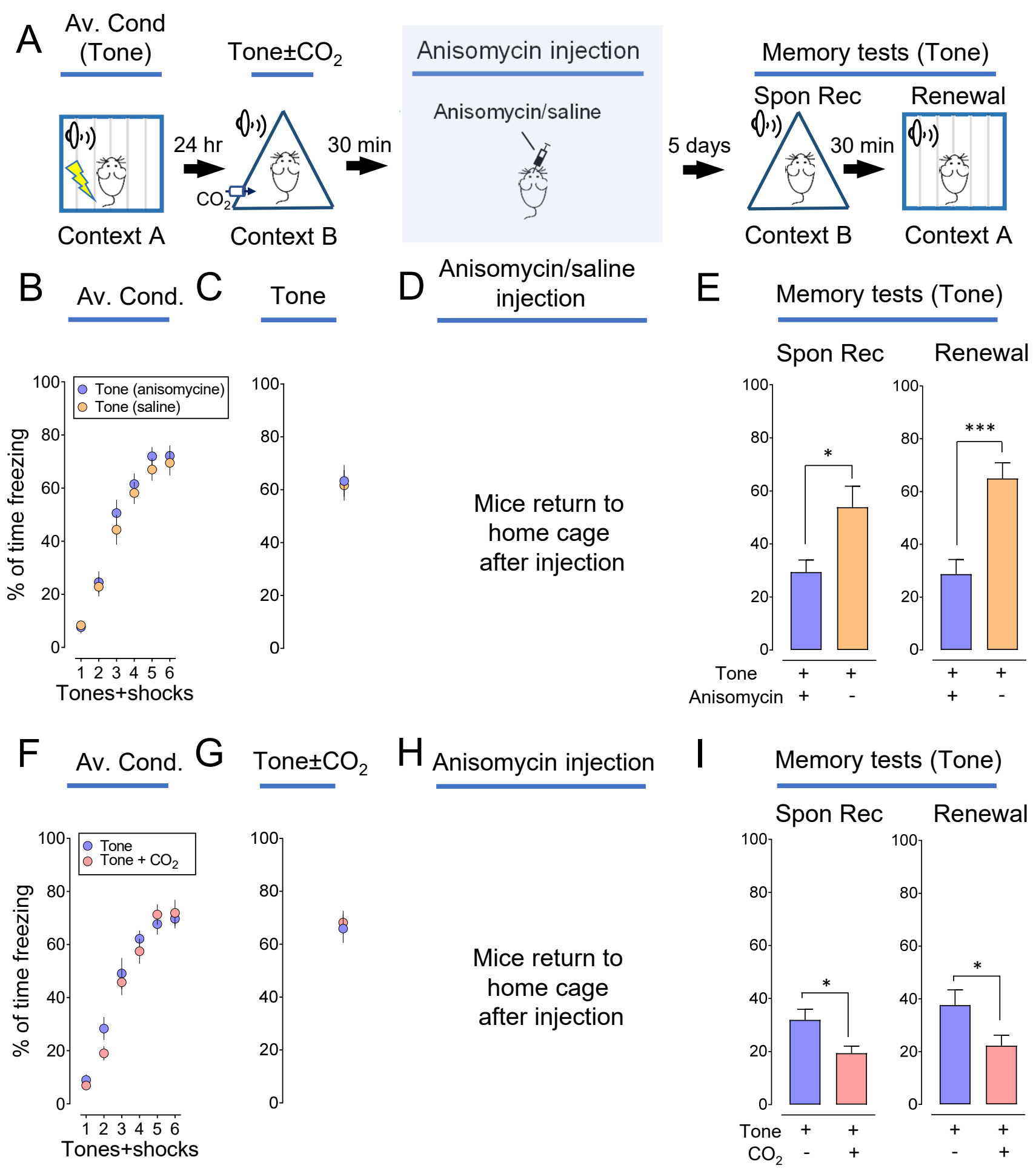
A Av. Cond. Tone, noise available under aCC-BY-NC-ND 4.0 International license.

Fig. 3 (Tone, noise) $\quad \pm \mathrm{CO}_{2}$ Extinction blocks (Tone) Memory tests (Tone, noise)

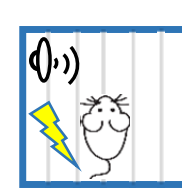

Context A

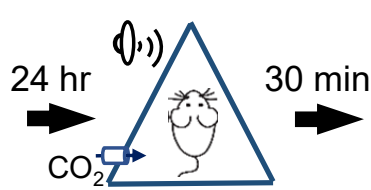

Context B

Ext 1

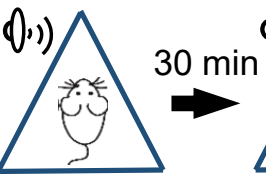

Context B
Ext 2

(0.1) 5 days

Spon Rec

Renewal

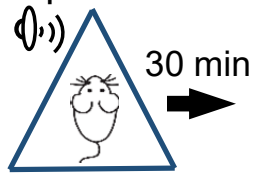

Context B
(-))
B

Av. Cond. Tone, Noise

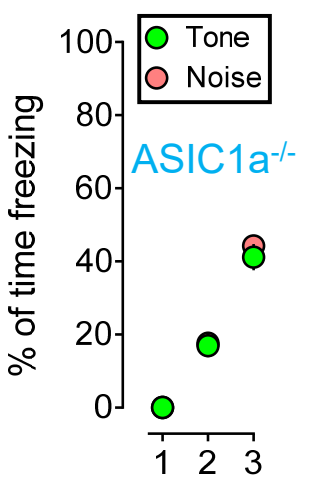

Tones+shocks
D
$E$ Memory tests (Tone, noise)
$\mathrm{F}$ Av. Cond.

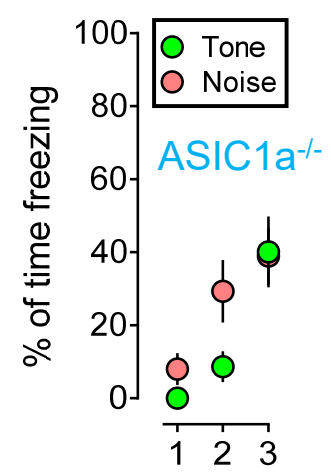

Tones+shocks

$\mathrm{J}$

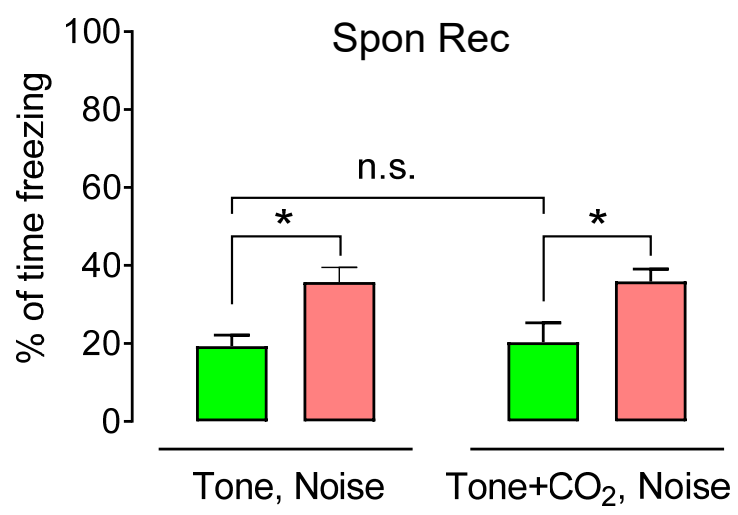

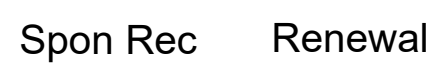
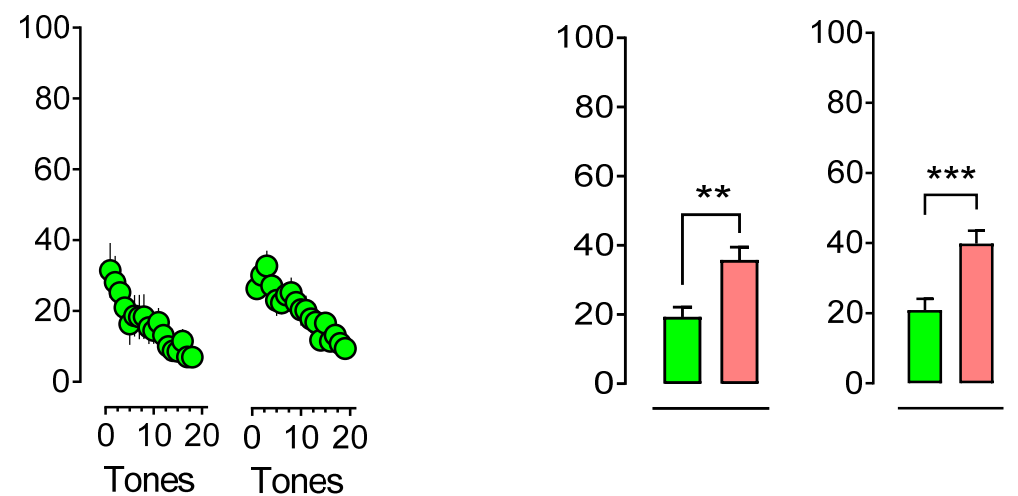
bioRxiv preprint doi: https://doi.org/10.1101/2021.01.04,425235; this version posted February 13, 2021. The copyright holder for this preprint (which was not certified by peer review) is the author/funder, who has granted bioRxiv a license to display the preprint in perpetuity. $y$ is made
(Tone) Tone $\pm \mathrm{CO}_{2} \quad$ Anisomycin injection

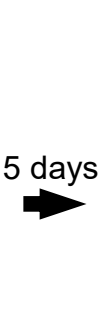
Memory tests (Tone)
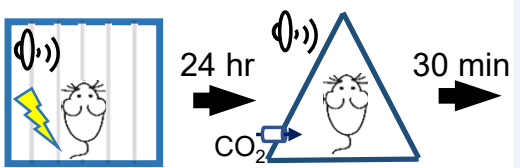
Anisomycin/saline
Context A
Context B

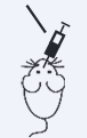
Spon Rec Renewal

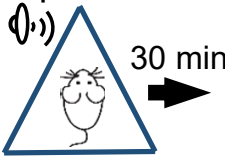
Context B
(0))

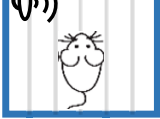

B Av. Cond. $\mathrm{C}_{\text {Tone } \pm \mathrm{CO}_{2}}$

D Anisomycin injection

E

Mice return to

home cage

after injection

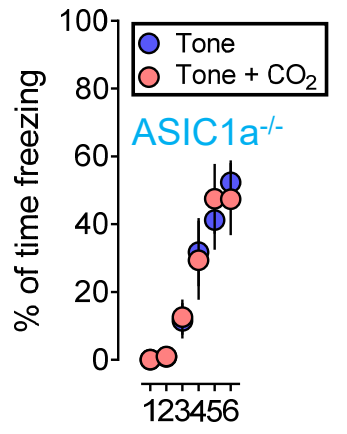

$\left.\begin{array}{r}100 \\ 80 \\ 60 \\ 40 \\ 20 \\ 0\end{array}\right]$

Tone+shocks

F Av. Cond.

Tone, noise

$\mathrm{F}_{\text {(Tone, noise) }} \pm \mathrm{CO}_{2}$

Anisomycin injection

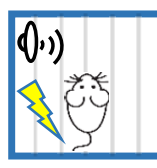

Context A

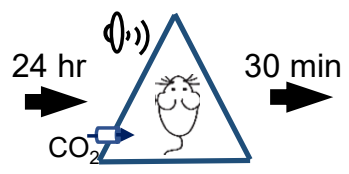

Context B

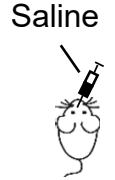

Mice return to home cage after injection

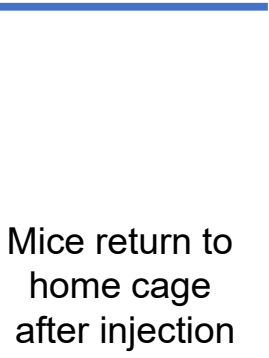

$G$ Av. Cond. $\mathrm{H}_{\text {Tone }}^{\mathrm{Noise}+\mathrm{CO}_{2}, \quad \mid \text { Anisomycin injection }}$

Memory tests (Tone, noise)

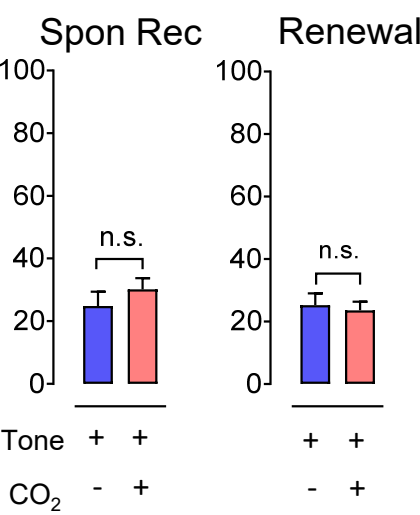

Spon Rec Renewal

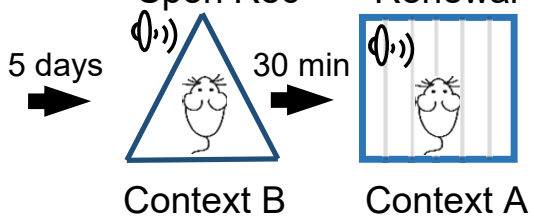

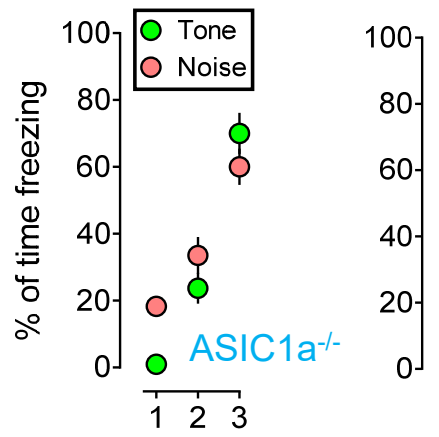

8

Tones+shocks

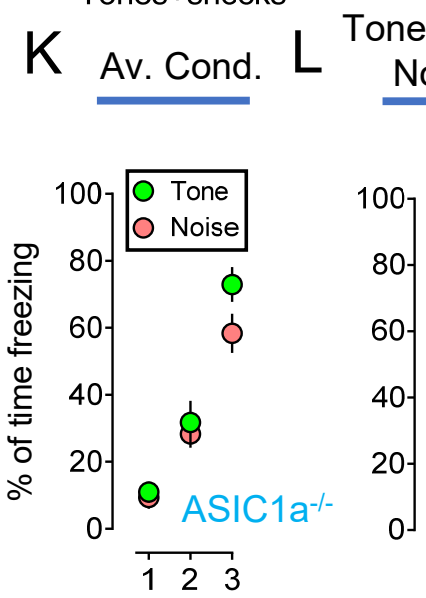

$M_{\text {Anisomycin injection }}$

N

Memory tests

Spon Rec Renewal

Mice return to

home cage

after injection

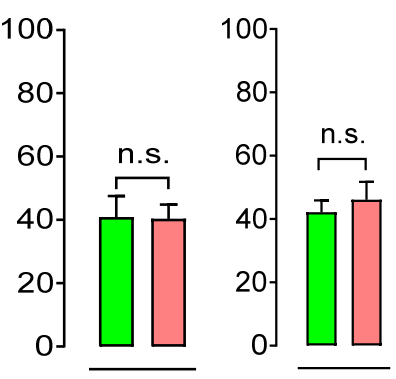


Fig. 5

A Av. Cond. Ret, $\pm \mathrm{CO}_{2} \quad$ Extinction blocks (Tone) Memory tests (Tone, noise) Context $\mathrm{A}$

B

Av. Cond.

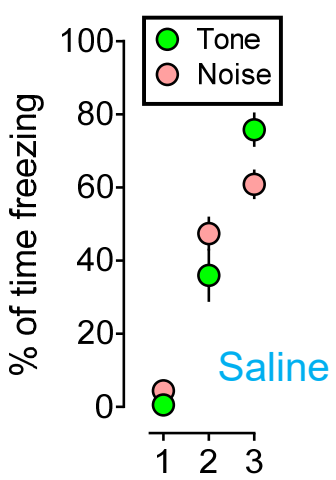

$\mathrm{F}$

Tones+shocks

Av. Cond.

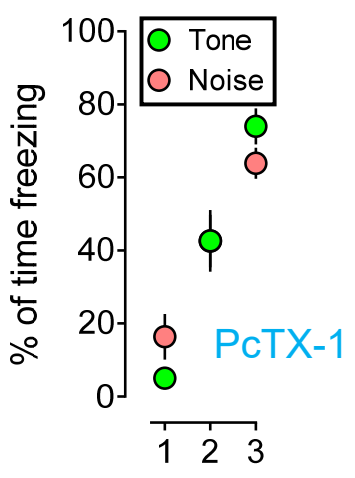

Tones+shocks

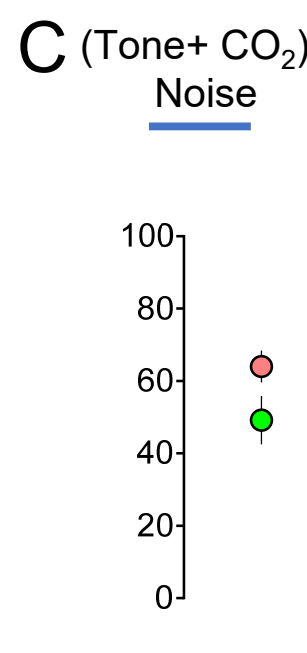

$\mathrm{G}\left(\mathrm{Tone}+\mathrm{CO}_{2}\right)$, Noise

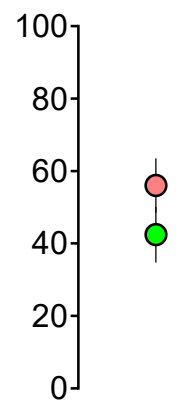

Spon Rec

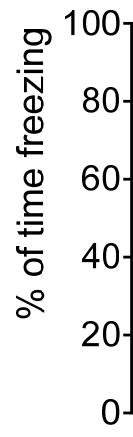

n.s.

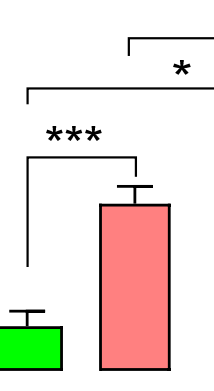

Saline
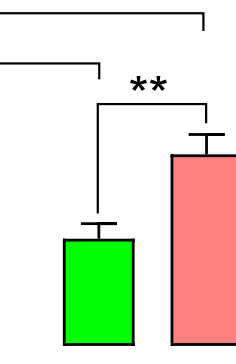

PcTX-1

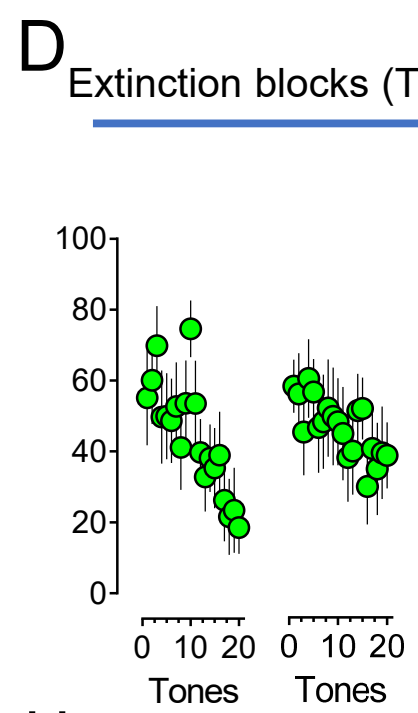

E
Memory tests (Tone, noise)

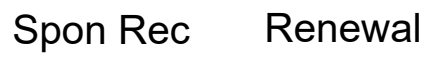

$\mathrm{H}$

Extinction blocks (Tone)

Memory tests (Tone, noise)
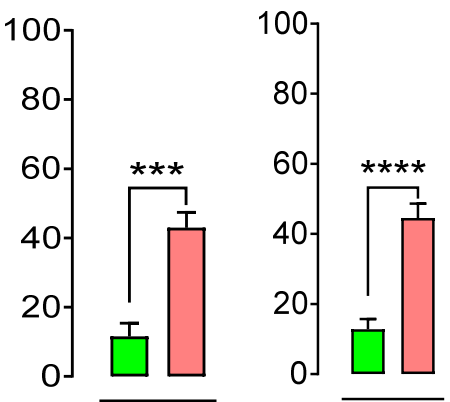

Spon Rec Renewal
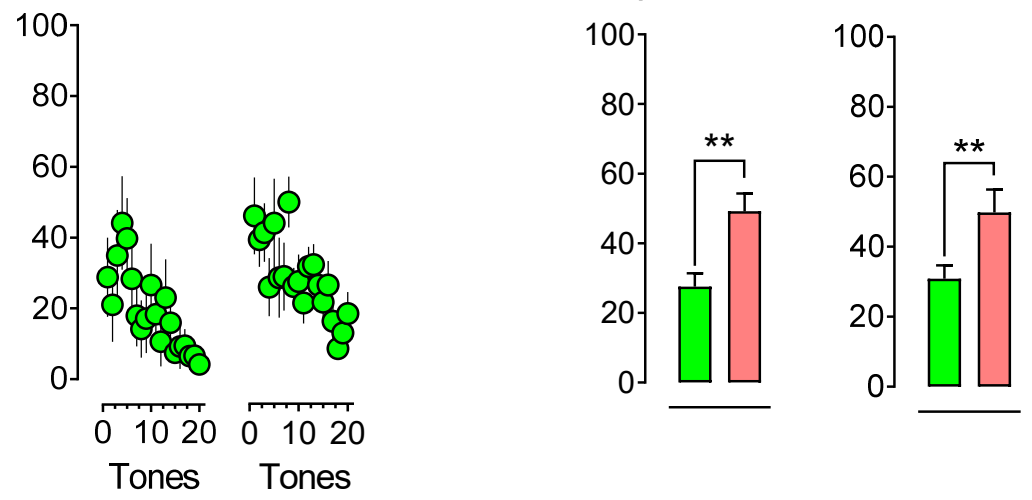

$\mathrm{K}$

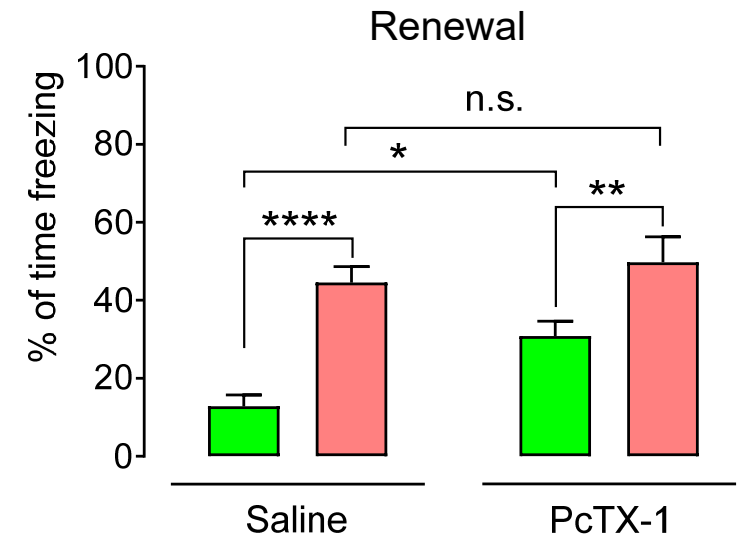




\section{Fig. 6}

A Av. Cond.

Tone or noise

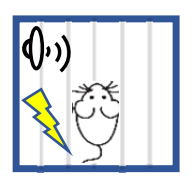

Context A

$\mathrm{B}$

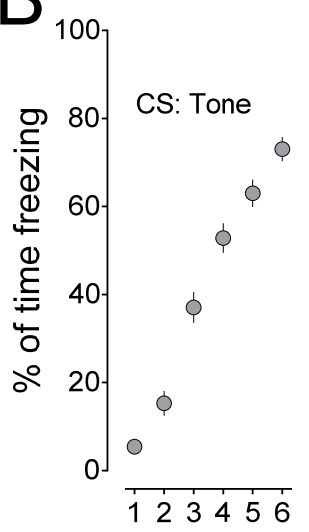

Tones+shocks

D

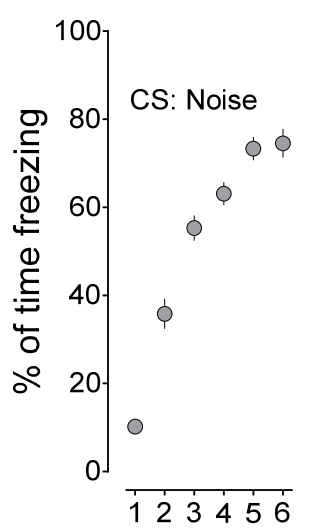

Tones+shocks

$\mathrm{F}$

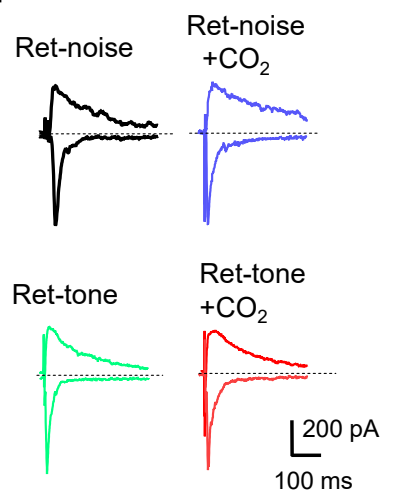

Ret $\pm \mathrm{CO}_{2}$
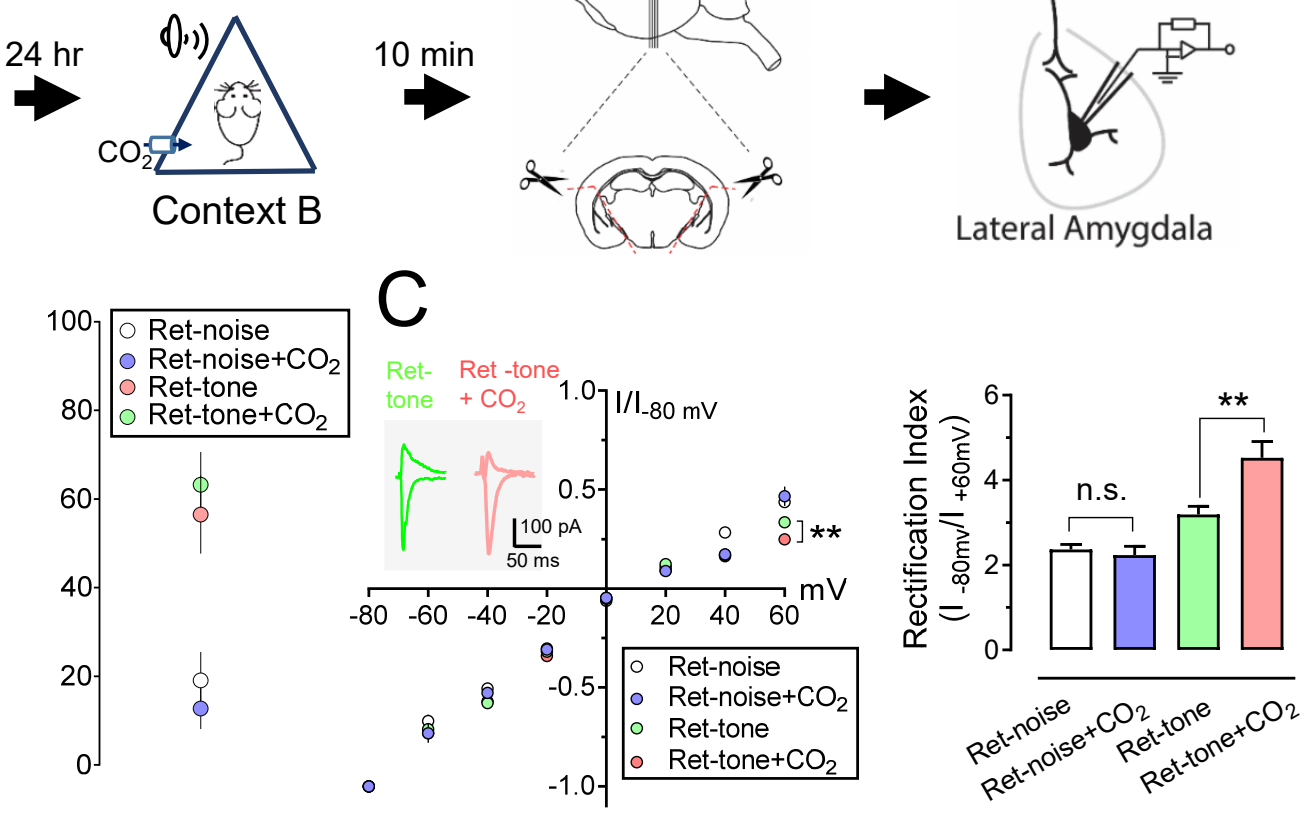

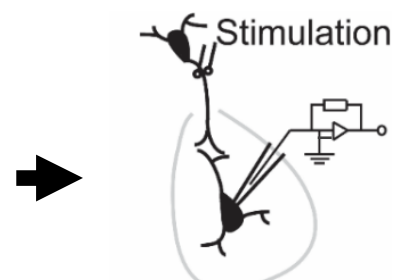

Lateral Amygdala 

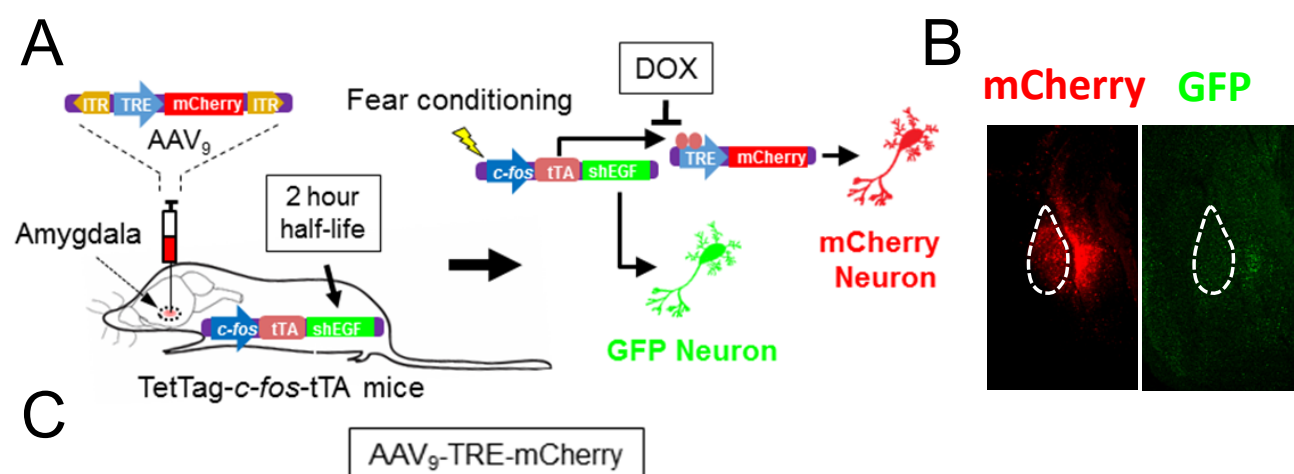

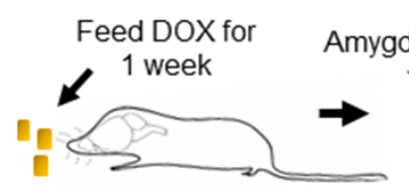

D

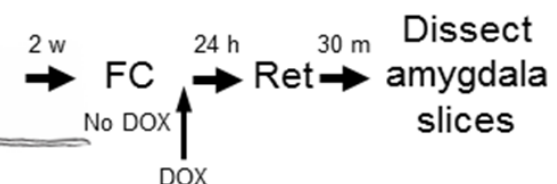

E
DAPI

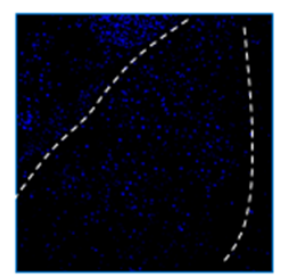

mCherry

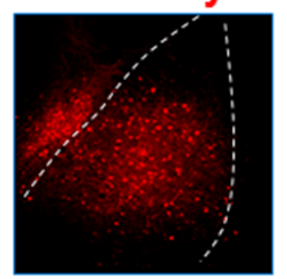

$G$

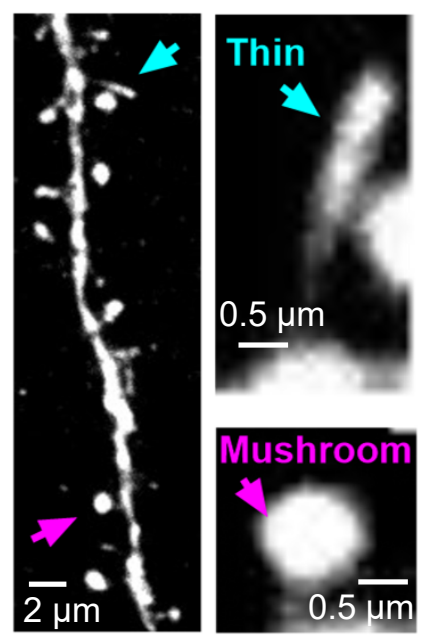

GFP
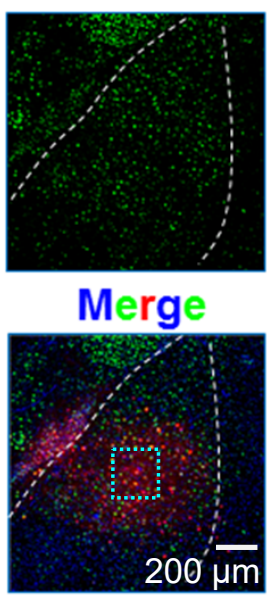

$\mathrm{H}$
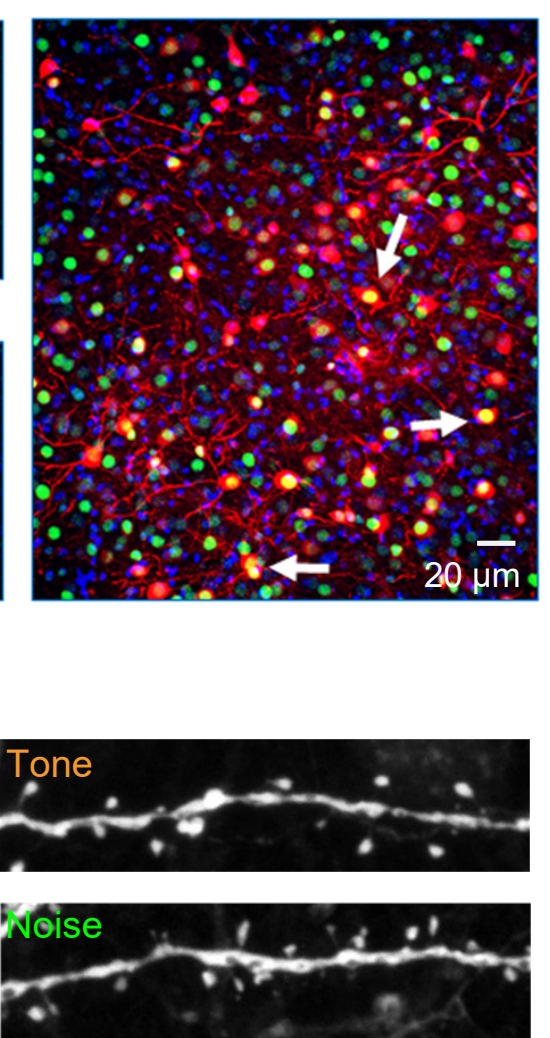

Mushroom spine

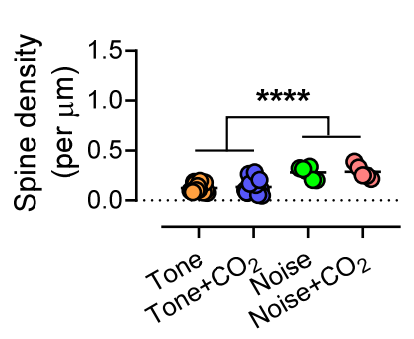

을 은

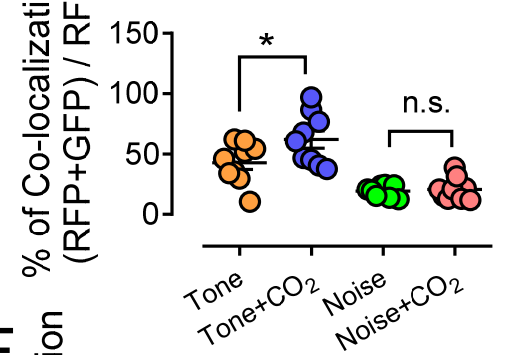

$F \stackrel{\frac{\delta}{ㅇ}}{\frac{0}{\pi}}$

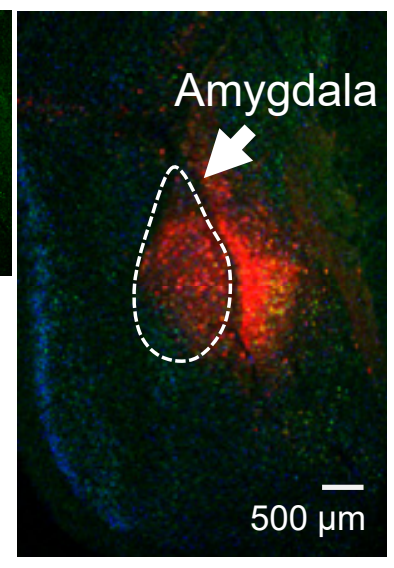

Merge
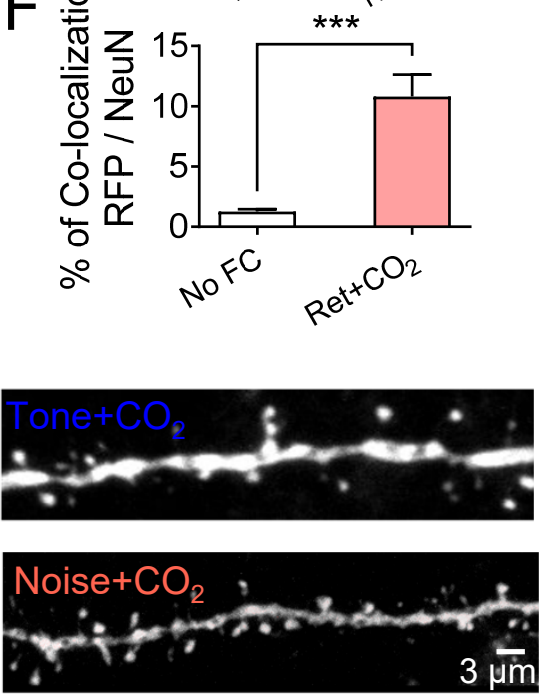

Thin spine

Total spine
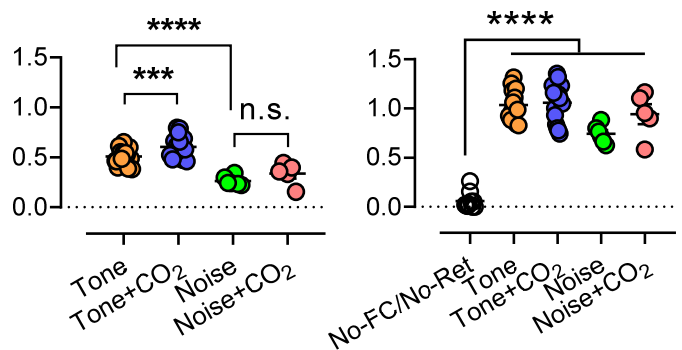
A Av. Cond.

Tone, noise Ret, $\pm \mathrm{CO}_{2} \quad$ Extinction blocks (noise) Memory tests (Tone, noise)

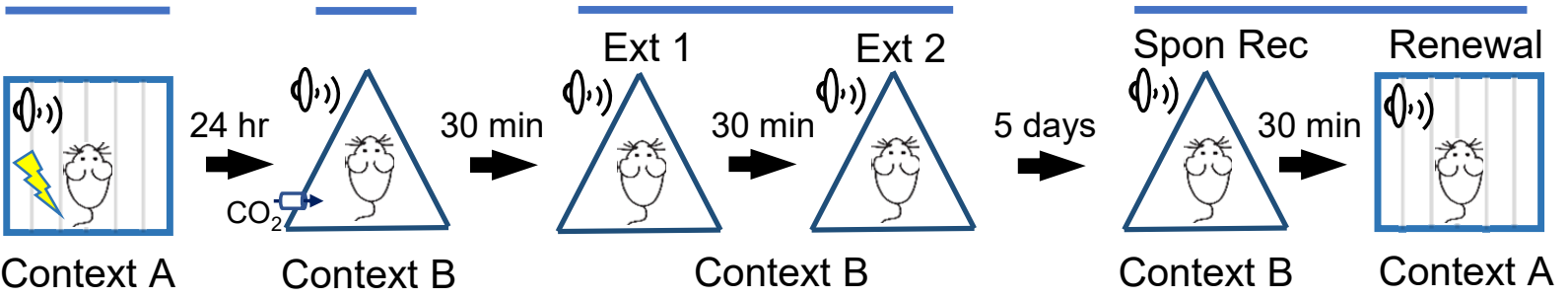

B

Av. Cond. ${ }_{\text {Tone, Noise }}$

$D_{\text {Extinction blocks (Noise) }} E_{\text {Memory tests (Tone, noise) }}$

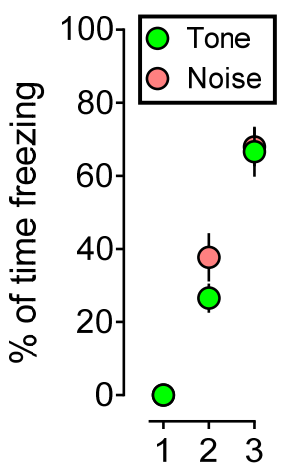
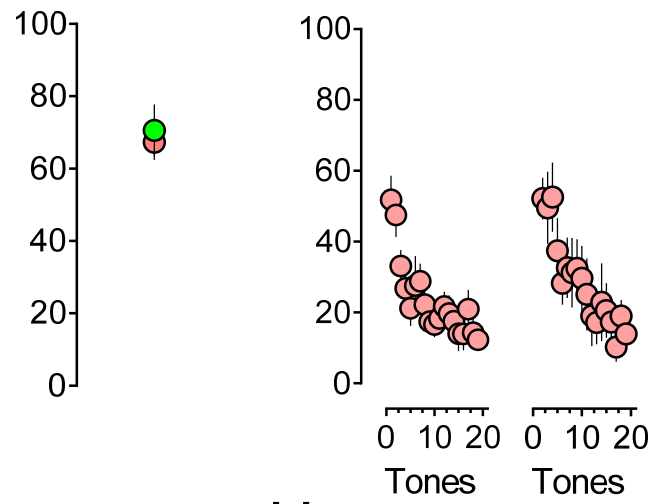

Spon Rec Renewal

F ${ }^{\text {Tones+shocks }} \mathrm{G}$

Tone,

Av. Cond. Noise $+\mathrm{CO}_{2}$

$\mathrm{H}$

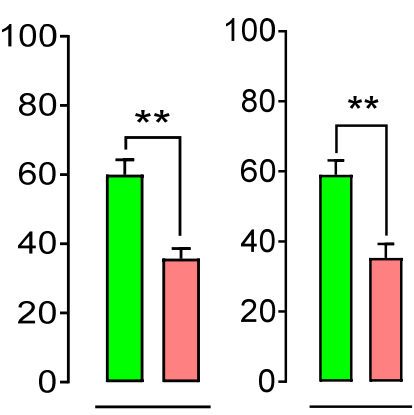

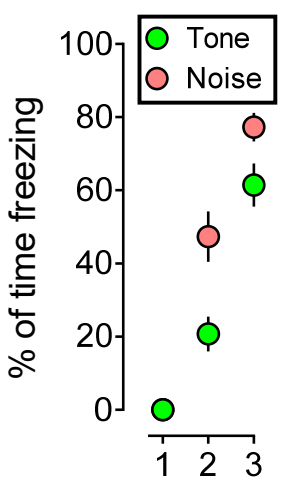

Tones+shocks

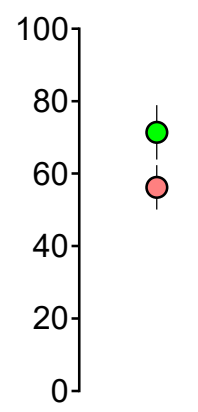

Spon Rec
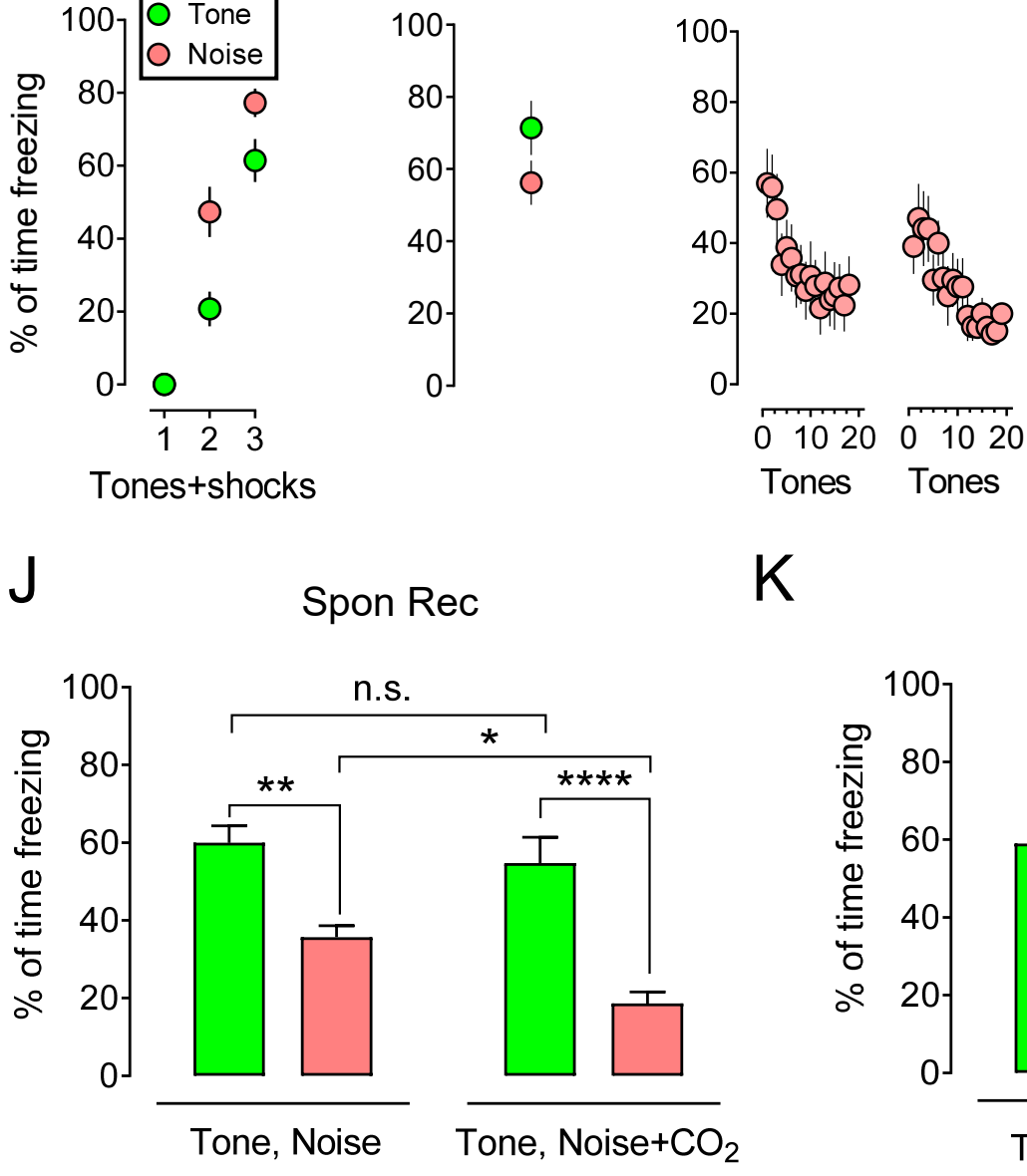

Memory tests (Tone, noise)
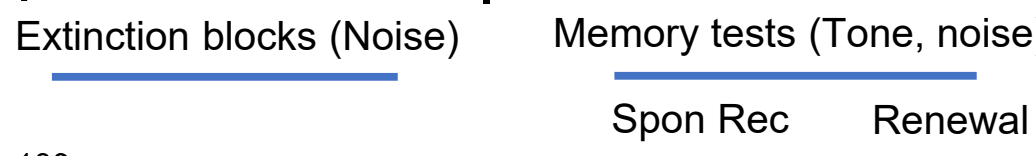

J

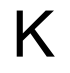

Renewal

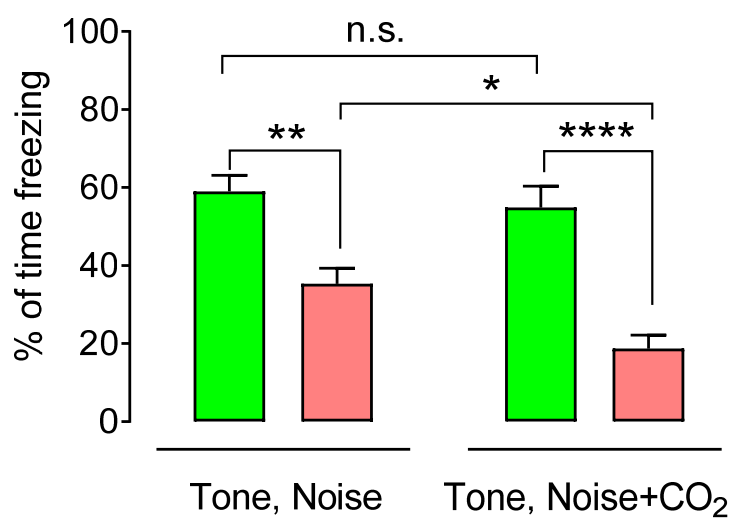




\section{Fig. s2}

A Av. Cond.

(Tone, noise) Ret, $\pm \mathrm{CO}_{2}$

\section{Extinction blocks}

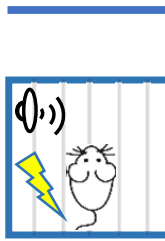

Context A

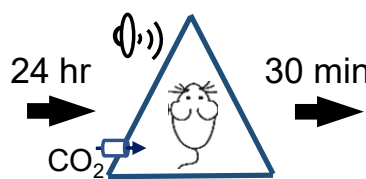

Context B
(Tone or noise)

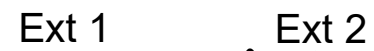

Memory tests (Tone, noise)

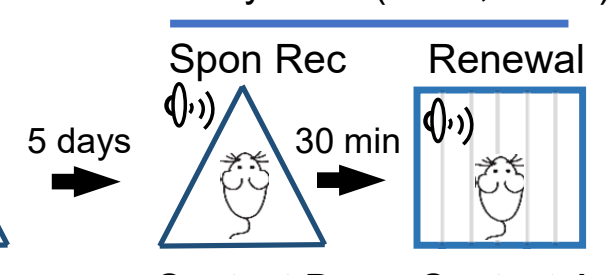

Context B Context A
B

Av. Cond.

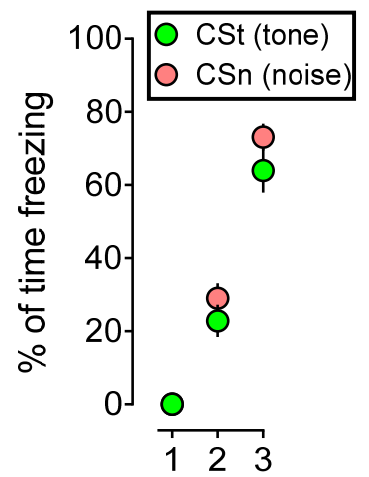

$F$

Tones+shocks

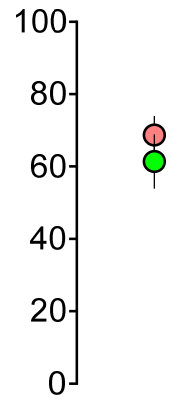

$\mathrm{G}\left(\right.$ Tone $\left.+\mathrm{CO}_{2}\right)$

Noise

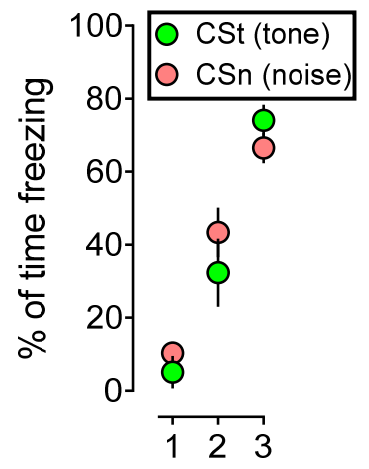

Tones+shocks

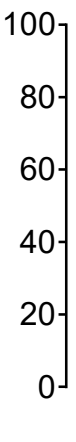

8

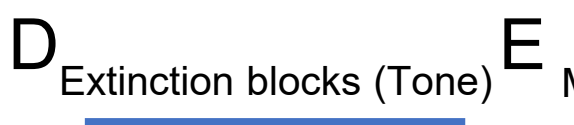

Context B

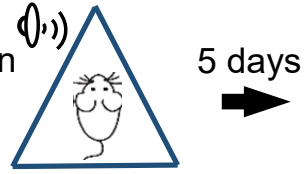

Memory tests (Tone, noise)

Spon Rec Renewal

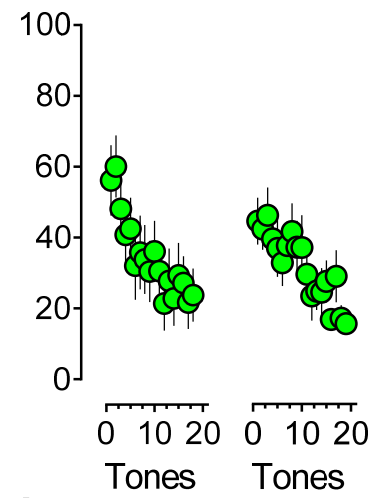

$\mathrm{H}$

Extinction blocks (Noise)

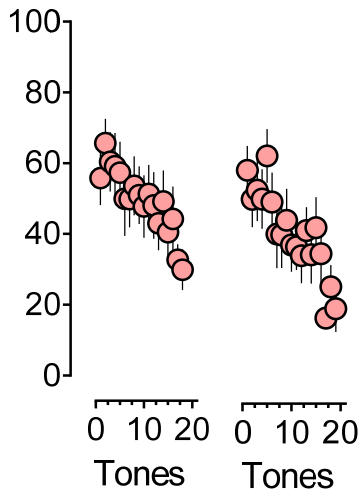

Memory tests (Tone, noise) Spon Rec Renewal

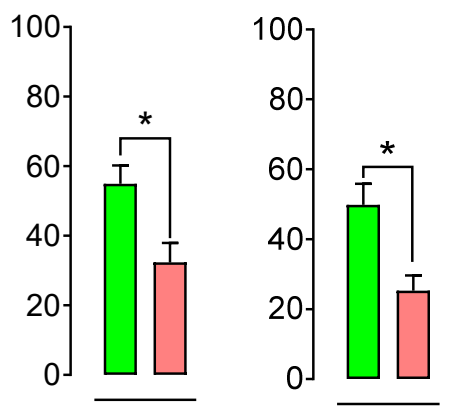


A

Av. Cond. Ret Anisomycin/saline injection

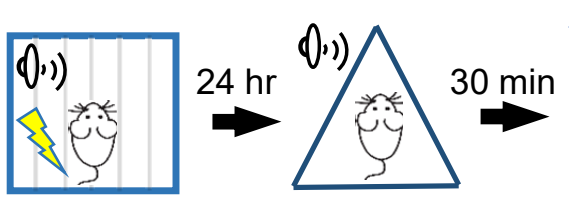

Context A

Context B
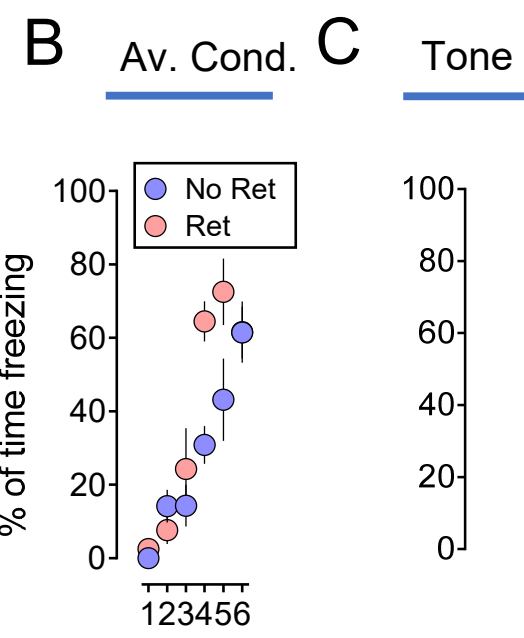

D Anisomycin injection

Anisomycin/saline

Tones+shocks

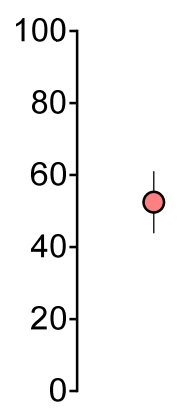

Mice return to

home cage

after injection
F Av. Cond. $G$ Tone, noise $H_{\text {Anisomycin injection }}$

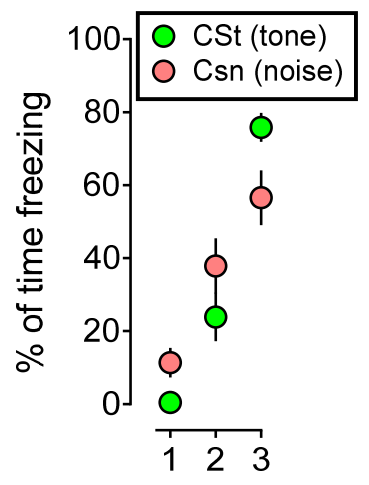

Mice return to home cage after injection

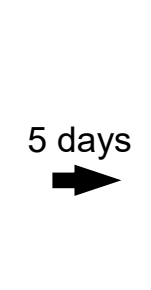

$\mathrm{E}$

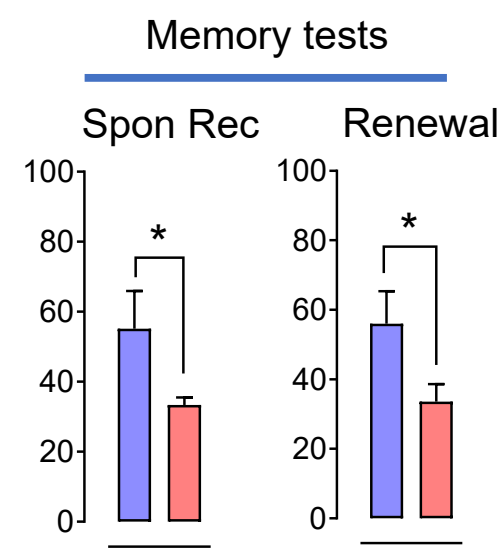

Memory tests

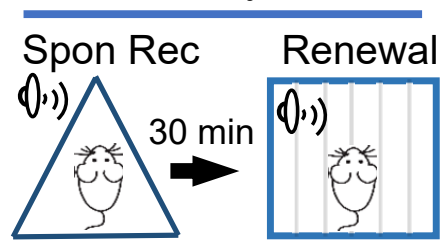

Context B Context A

Tones+shocks

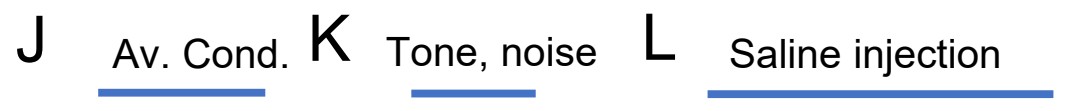

M
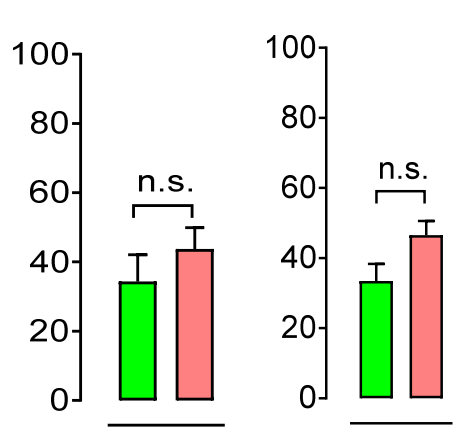

Memory tests

Spon Rec Renewal
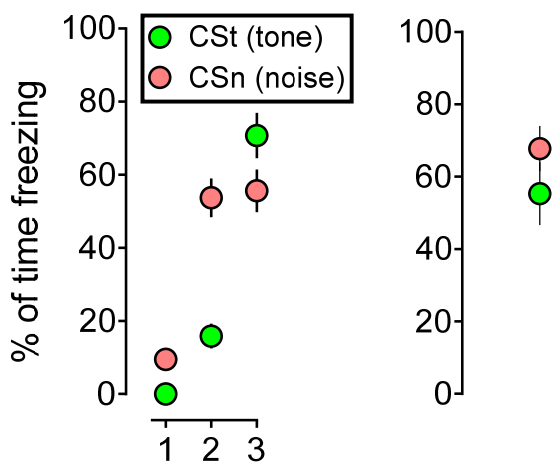

Mice return to home cage after injection

\section{Memory tests}

Spon Rec Renewal

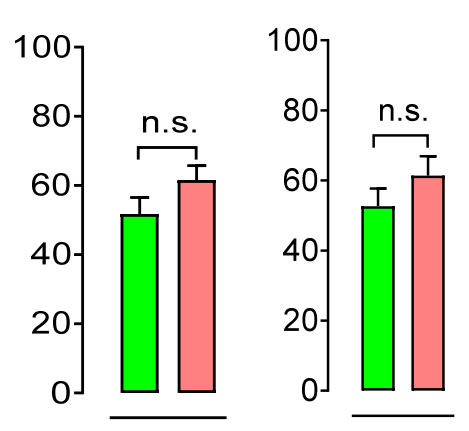




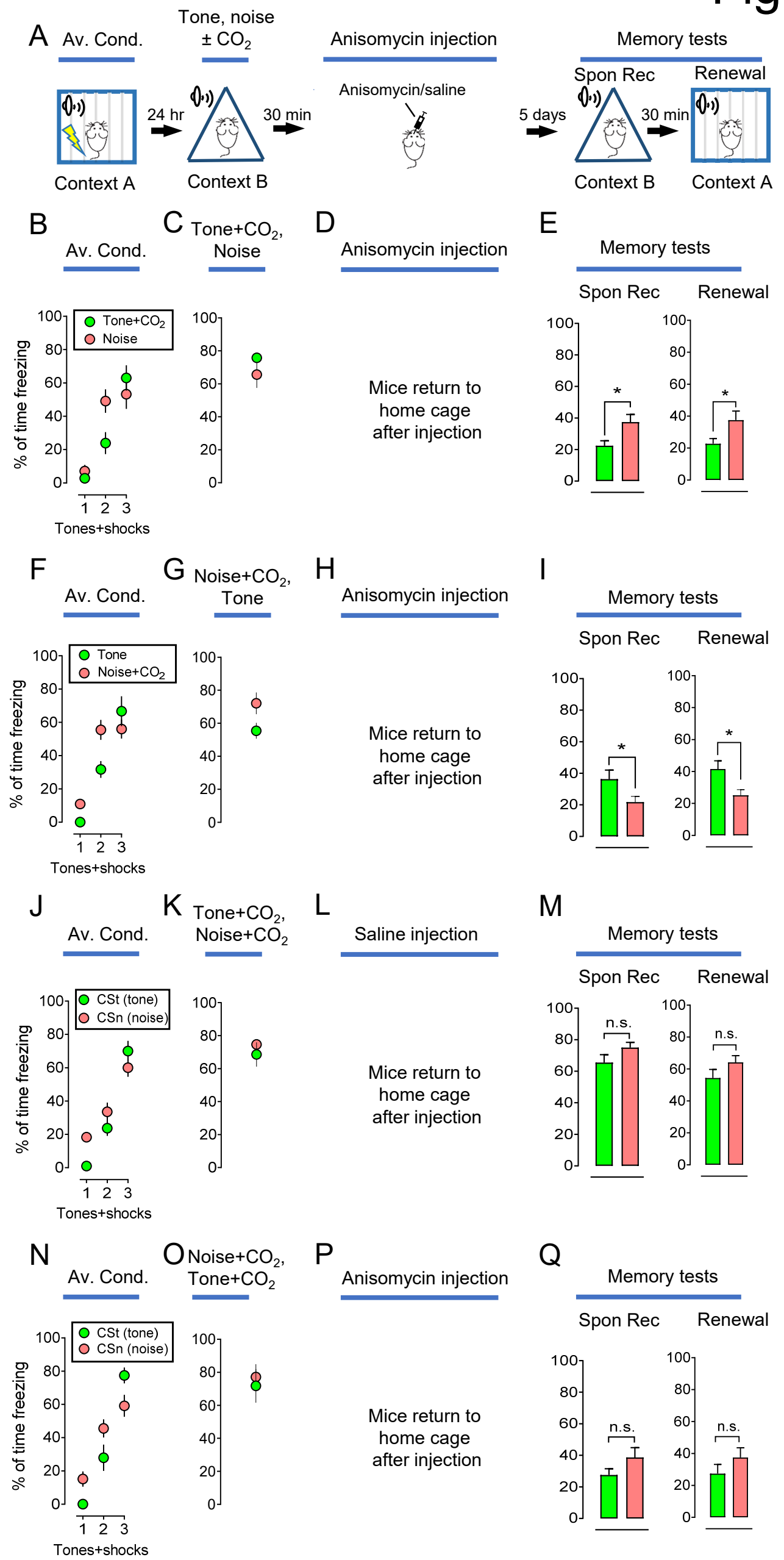

Fig. s4 


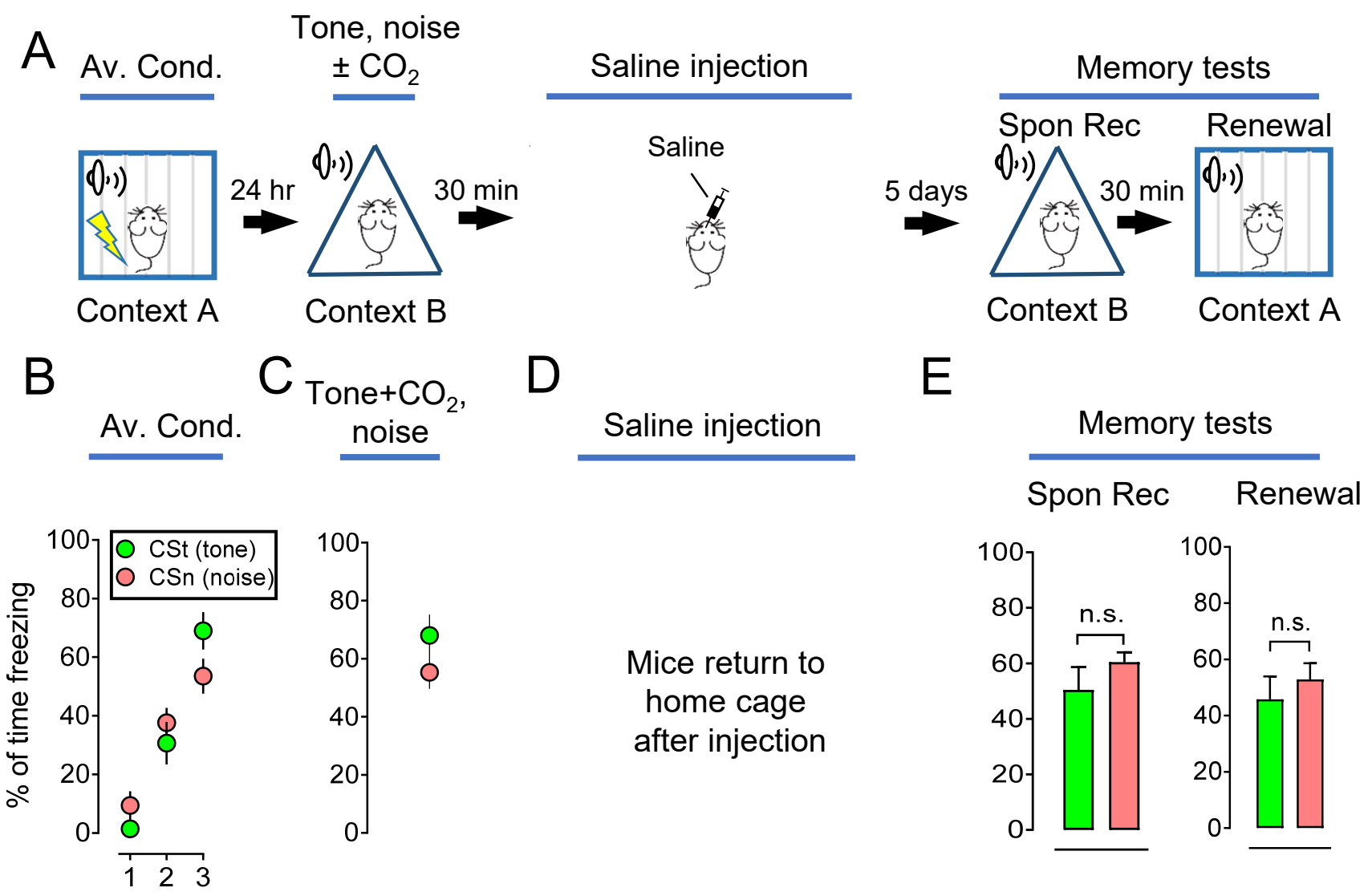

Tones+shocks

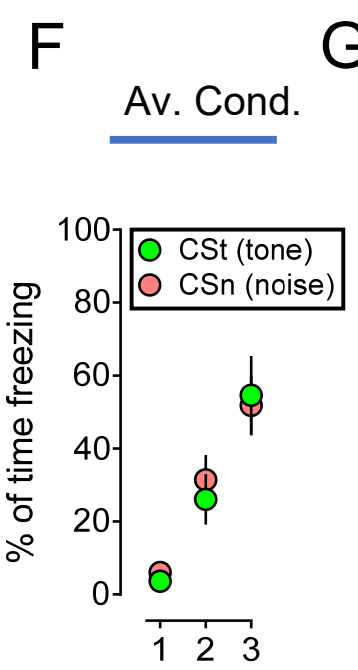

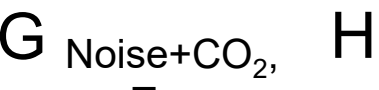

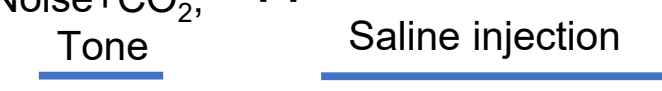

Mice return to home cage after injection
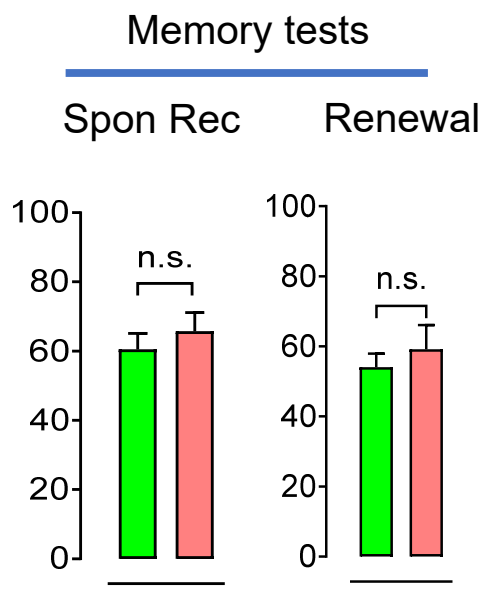

Tones+shocks 